\title{
Anomalous transport from holography: part II
}

\author{
Yanyan Bu ${ }^{1, \mathrm{a}}$, Michael Lublinsky ${ }^{1,2, \mathrm{~b}}$, Amir Sharon ${ }^{1, \mathrm{c}}$ \\ ${ }^{1}$ Department of Physics, Ben-Gurion University of the Negev, Beersheba 84105, Israel \\ ${ }^{2}$ Physics Department, University of Connecticut, 2152 Hillside Road, Storrs, CT 06269-3046, USA
}

Received: 24 February 2017 / Accepted: 15 March 2017 / Published online: 27 March 2017

(C) The Author(s) 2017. This article is an open access publication

\begin{abstract}
This is a second study of chiral anomaly-induced transport within a holographic model consisting of anomalous $U(1)_{V} \times U(1)_{A}$ Maxwell theory in Schwarzschild$\mathrm{AdS}_{5}$ spacetime. In the first part, chiral magnetic/separation effects (CME/CSE) are considered in the presence of a static spatially inhomogeneous external magnetic field. Gradient corrections to CME/CSE are analytically evaluated up to third order in the derivative expansion. Some of the third order gradient corrections lead to an anomaly-induced negative $B^{2}$-correction to the diffusion constant. We also find modifications to the chiral magnetic wave nonlinear in $B$. In the second part, we focus on the experimentally interesting case of the axial chemical potential being induced dynamically by a constant magnetic and time-dependent electric fields. Constitutive relations for the vector/axial currents are computed employing two different approximations: (a) derivative expansion (up to third order) but fully nonlinear in the external fields, and (b) weak electric field limit but resuming all orders in the derivative expansion. A non-vanishing nonlinear axial current (CSE) is found in the first case. The dependence on magnetic field and frequency of linear transport coefficient functions is explored in the second.
\end{abstract}

\section{Introduction and summary}

Fluid dynamics $[1,2]$ is an effective long-wavelength description of most classical or quantum many-body systems at nonzero temperature. It is defined in terms of constitutive relations, which relate thermal expectation values of conserved currents to thermodynamical variables and external fields. The derivative expansion in fluid-dynamic variables such as the velocity or charge densities accounts for deviations from thermal equilibrium. At each order, the deriva-

\footnotetext{
a e-mail: yybu@post.bgu.ac.il

be-mail: lublinm@bgu.ac.il

c e-mail: sharon.amir24@gmail.com
}

tive expansion is fixed by thermodynamic considerations and symmetries, up to a finite number of transport coefficients, such as the viscosity, diffusion constant and conductivity. The latter are not calculable from hydrodynamics itself, but have to be determined from underlying microscopic theory or experimentally.

Although fluid dynamics has a long history, the theoretical foundations of relativistic viscous hydrodynamics are not yet fully established. The Navier-Stokes hydrodynamics leads to violation of causality: the set of fluid dynamical equations makes it possible to propagate signals faster than light. To overcome this problem, simulations of relativistic hydrodynamics are usually based on phenomenological prescriptions of [3-6], which admix viscous effects from second order derivatives, so as to make the fluid dynamical equations causal. References [3-6] introduced retardation effects for irreversible currents, which, via the equations of motion, become additional degrees of freedom. In other words, one needs to include higher order gradient terms in the derivative expansion in order to obtain a causal formulation. In general, causality is violated if the derivative expansion is truncated at any fixed order. It is supposed to be restored when all-order gradient terms are included, which we refer to as all-order resummed hydrodynamics. Resummed hydrodynamics is UV complete in a sense that it has a well-defined large frequency/momenta limit. Yet it is an effective theory of hydrodynamic variables only, ${ }^{1}$ which emerges after most of the degrees of freedom of the underlying microscopic theory are integrated out.

The most general parity-even linear in external fields and charge density off-shell constitutive relation for a vector current has the following form:

$J^{t}=\rho, \vec{J}=-\mathcal{D} \vec{\nabla} \rho+\sigma_{e} \vec{E}+\sigma_{m} \vec{\nabla} \times \vec{B}$,

\footnotetext{
${ }^{1}$ In fact there are infinitely many such variables (see Ref. [7] for a discussion).
} 
where $\rho$ is a vector charge density and the diffusion $\mathcal{D}$, electric/magnetic conductivities $\sigma_{e / m}$ are functionals of spacetime derivatives. In terms of hydrodynamic expansion, the constitutive relation (1) provides all-order resummation of gradients of the fluid-dynamic variables (the charge density $\rho$ ) and external fields $(\vec{E}$ and $\vec{B})$. In momentum space $\mathcal{D}$ and $\sigma_{e / m}$ are functions of frequency $\omega$ and momentum squared $q^{2}$ (assuming isotropic medium), which we refer to as transport coefficient functions (TCFs). Via the inverse Fourier transform, TCFs appear as memory functions in the constitutive relation [8].

For a holographic charged plasma dual to $U(1)$ Maxwell theory in Schwarzschild-AdS 5 TCFs were studied in depth in [9]. The derivative resummation in the constitutive relation was implemented via the technique of [7,10-12], which was originally invented to resum all-order velocity gradients (linear in the velocity amplitude) in the energy-momentum tensor of a holographic conformal fluid. ${ }^{2}$ It is important to stress that this linearisation procedure is a mathematically well-controlled approximation: the perturbative expansion corresponds to a formal expansion in the amplitudes of fluiddynamic variables and external fields, without any additional assumptions. In this respect, the implemented approximation is identical to that of the linear response theory based on twopoint correlators.

Our technique follows closely the original idea of [14], which relates fluid's constitutive relations for the boundary theory to solving equations of motion in the bulk. However, an important new element of our formalism is that it is not based on current conservation (i.e., "off-shell" formalism), which makes it essentially different from the "onshell" formalism of [14]. Constitutive relations and TCFs can be uniquely determined from dynamical components of the bulk equations only, while the constraint component in the bulk is equivalent to continuity equation on the boundary.

Chiral anomalies emerge and play an important role in relativistic QFTs with massless fermions. The anomaly is reflected in three-point functions of currents associated with global symmetries. When the global $U(1)$ currents are coupled to external electromagnetic fields, the triangle anomaly renders the axial current non-conserved,

$\partial_{\mu} J^{\mu}=0, \quad \partial_{\mu} J_{5}^{\mu}=12 \kappa \vec{E} \cdot \vec{B}$,

where $J^{\mu} / J_{5}^{\mu}$ are vector/axial currents, and $\kappa$ is an anomaly coefficient. For $S U\left(N_{\mathrm{c}}\right)$ gauge theory with a massless Dirac

\footnotetext{
${ }^{2}$ One might be concerned that the hydrodynamic derivative expansion forms an asymptotic series with zero radius of convergence [13]. However, contrary to our linearised study, this conclusion applies to nonlinear hydrodynamics in which the number of terms grows factorially with the number of gradients. What is more important is that our approach does not rely on explicit resummation of the gradient series and thus is safe from any convergence related uncertainties.
}

fermion in fundamental representation, $\kappa=e N_{\mathrm{c}} /\left(24 \pi^{2}\right)$, and $e$ is an electric charge which below will be set to unit.

The presence of triangle anomalies requires a modification of the usual constitutive relations for the currents. An example of such a modification is the chiral magnetic effect (CME) $[15-19],{ }^{3}$ that is, the induction of an electric current along the applied magnetic field. CME relies on chiral imbalance, which is usually parameterised by an axial chemical potential. Studies of CME can be found in e.g. [23-29] based on perturbation theory, in e.g. [30-35] within lattice simulations, and in e.g. [36-50] for the strongly coupled regime based on the AdS/CFT correspondence [51-53].

The chiral separation effect (CSE) $[54,55]$ is another interesting phenomenon induced by the anomalies. It is reflected in a separation of the chiral charges along an external magnetic field at finite density of vector charges. Chiral charges can also be separated along external electric field, when both vector and axial charge densities are nonzero, the so-called chiral electric separation effect (CESE) [56,57].

In heavy ion collisions, experimentally observable effects induced by the anomalies were discussed in [58-62]. We refer the reader to [63-67] and the references therein for comprehensive reviews of the subject of anomalous transports.

In [68] we went beyond [9] focussing on transport properties induced by the chiral anomaly. The holographic model was modified to be anomalous $U(1)_{V} \times U(1)_{A}$ Maxwell theory in the Schwarzschild-AdS ${ }_{5}$ case. Under various approximations, off-shell constitutive relations were derived for vector/axial currents. In a weak external field approximation, all-order derivatives in the vector/axial currents were resummed into six momenta-dependent TCFs: the diffusion, the electric/magnetic conductivity, and three anomaly-induced TCFs. The latter generalise the chiral magnetic/separation effects. Beyond weak external field approximation, nonlinear transports were also revealed when constant background external fields are present. Particularly, the chiral magnetic effect, including all-order nonlinearity in magnetic field, was proven to be exact when all external fields except for a constant magnetic field are turned off. Nonlinear corrections to the currents' constitutive relations due to electric and axial external fields were computed.

In the present work we continue the study of anomalyinduced transports within the holographic model of [68]. No axial external fields will be turned on in this work. As in $[9,68]$ we work in the probe limit so that the currents and energy-momentum tensor decouple. In dual gravity, the probe limit ignores the back-reaction of the gauge dynamics on the geometry. The holographic model under study consists of two Maxwell fields in the Schwarzschild-AdS 5 black brane geometry. The chiral anomaly is holographi-

\footnotetext{
${ }^{3}$ See also [20-22] for earlier, related work.
} 
cally realised via the gauge Chern-Simons actions for both Maxwell fields. Such a holographic setup can be realised via a top-down brane construction of $D 4 / D 8 / \overline{D 8}$ [69].

Before diving into the details presented in the following sections, we summarise our main results. The paper is split into two largely independent parts. In the first part, we consider a setup in which a static but spatially varying magnetic field is the only external field that is turned on. Then the constitutive relations for the vector/axial currents are

$$
\begin{aligned}
& J^{t}=\rho, \quad J^{i}=-\frac{1}{2} \partial_{i} \rho+\underline{12 \kappa \mu_{5} B_{i}}-G_{i}(x=\infty), \\
& J_{5}^{t}=\rho_{5}, \quad J_{5}^{i}=-\frac{1}{2} \partial_{i} \rho_{5}+\underline{12 \kappa \mu B_{i}}-H_{i}(x=\infty),
\end{aligned}
$$

where $\rho / \rho_{5}$ are vector/axial charge densities, the underlined terms in $J^{i} / J_{5}^{i}$ are the chiral magnetic/separation effects. $G_{i}, H_{i}$ contain derivatives of $\rho, \rho_{5}, \vec{B}$ and are defined in Sect. 3. It is important to stress that, in contrast to the above discussion of linearised hydro, (3) and (4) are exact, without any approximations for $\rho, \rho_{5}, \vec{B}$. The nonlinearity of the CME/CSE in external magnetic field $\vec{B}$ is completely accounted for by the chemical potentials $\mu, \mu_{5}$. The non-derivative part of (3) is consistent with the "nonrenormalisability" of CME [48,49,70,71]. However, as will be clear from (48) and (49), the derivative corrections in $G_{i}, H_{i}$ introduce new effects, which do modify the original CME. Particularly, the currents along the direction of $\mathbf{B}$ get affected.

When $\rho, \rho_{5}, \vec{B}$ vary slowly from point to point, $G_{i}, H_{i}$ can be calculated order-by-order within boundary derivative expansion. Let us introduce a scaling parameter $\lambda$ :

$\partial_{\mu}=\left(\partial_{t}, \partial_{i}\right) \longrightarrow\left(\lambda \partial_{t}, \lambda \partial_{i}\right)$.

Then derivative counting goes by powers of $\lambda$. Up to second order in derivative expansion, we calculated $G_{i}, H_{i}$ and chemical potentials $\mu, \mu_{5}$ without any further assumptions. Given that these results are rather lengthy, we postpone to present them in Sect. 3; see (48) and (49) and (50). At third order $\mathcal{O}\left(\partial^{3}\right)$, for $G_{i}, H_{i}$ we calculated only terms that are linear in $\rho, \rho_{5}$; see (51) and (52) for a complete listing. Among these third order terms, the diffusion constant $\mathcal{D}_{0}$ (i.e., the DC limit of the diffusion function $\mathcal{D}$ ) gets a negative $B$-dependent correction

$\mathcal{D}_{0}=\frac{1}{2}-18(2 \log 2-1) \kappa^{2} B^{2}$.

To the best of our knowledge, this is the first anomaly-induced correction to the diffusion constant, and, being negative, it happens to violate the universal form of [72].

With the third order results for $J^{\mu}$ and $J_{5}^{\mu}$, we also computed the dispersion relation for a free mode that can propagate in the medium:

$$
\begin{aligned}
\omega= & {\left[\mp 1+36(1-2 \log 2) \kappa^{2} \mathbf{B}^{2}\right] 6 \kappa \vec{q} \cdot \overrightarrow{\mathbf{B}} } \\
& -\left[\frac{1}{2}+18(1-2 \log 2) \kappa^{2} \mathbf{B}^{2}\right] i q^{2}-\frac{i}{8} q^{4} \log 2+\cdots,
\end{aligned}
$$

where $\overrightarrow{\mathbf{B}}$ means a constant magnetic field. The first term in (7) represents the chiral magnetic wave (CMW) [70]. Interestingly, we see nonlinear in $\mathbf{B}$ corrections to both the speed of CMW and its decay rate. Note that we also expect the emergence in (7) of the following terms: $(\vec{q} \cdot \overrightarrow{\mathbf{B}})^{2}, q^{2}(\vec{q} \cdot \overrightarrow{\mathbf{B}})$, $(\vec{q} \cdot \overrightarrow{\mathbf{B}})^{3}, q^{2}(\vec{q} \cdot \overrightarrow{\mathbf{B}})^{2}$ and $(\vec{q} \cdot \overrightarrow{\mathbf{B}})^{4}$. However, our ability to determine coefficients of these terms is limited by the undertaken approximations.

In the second part of this work, we focus on a special setup which is experimentally accessible in condensed matter systems. ${ }^{4}$ CME emerges from a nonzero axial chemical potential $\mu_{5}$, which is usually assumed to have some background profile. It is, however, possible to induce $\rho_{5}$ (and thus $\mu_{5}$ ) dynamically through the interplay between the electric and magnetic fields, as is clear from the continuity equation (2). Specifically, we are ready to consider a constant magnetic field $\overrightarrow{\mathbf{B}}$ and a time-dependent but spatially homogeneous electric field $\vec{E}(t)$. For simplicity the charge densities $\rho, \rho_{5}$ will be assumed to be spatially homogeneous too ${ }^{5}$. From (2), $\rho$ could be set zero. The constitutive relations for the vector/axial currents are

$$
\begin{aligned}
J^{t}=0, \quad J^{i}= & E_{i}+\partial_{t} E_{i}+12 \kappa \mu_{5} \mathbf{B}_{i} \\
& -12 \kappa \epsilon^{i j k} \mathbb{A}_{j}(1) E_{k}+\bar{G}_{i}(x=\infty), \\
J_{5}^{t}=\rho_{5}, \quad J_{5}^{i}= & 12 \kappa \mu \mathbf{B}_{i}-12 \kappa \epsilon^{i j k} \mathbb{V}_{j}(1) E_{k} \\
& +\bar{H}_{i}(x=\infty),
\end{aligned}
$$

where $\mathbb{V}_{j}(1), \mathbb{A}_{j}(1), \bar{G}_{i}$ and $\bar{H}_{i}$ depend on $\rho_{5}, \vec{E}$ and $\overrightarrow{\mathbf{B}}$ nonlinearly and will be computed below. Our study is further split into two parts. In Sect. 4.1, $\mathbb{V}_{j}(1), \mathbb{A}_{j}(1), \bar{G}_{i}$ and $\bar{H}_{i}$ will be evaluated perturbatively within the gradient expansion (5). These perturbative results can be found in (65)-(68). In Sect. 4.2 , we will consider another approximation-linearisation of the constitutive relations in the external electric field.

In the linearised regime, we assume the following scaling for $\rho_{5}, \vec{E}$ and $\overrightarrow{\mathbf{B}}$ :

$\rho_{5} \sim \mathcal{O}(\epsilon), \quad \vec{E}(t) \sim \mathcal{O}(\epsilon), \quad \overrightarrow{\mathbf{B}} \sim \mathcal{O}\left(\epsilon^{0}\right)$,

which will be referred to as an amplitude expansion. To linear order in $\epsilon$, the vector/axial currents are

$$
\begin{aligned}
J^{t}=0, \quad \vec{J} & =\sigma_{e} \vec{E}+\tau_{1} \kappa \rho_{5} \overrightarrow{\mathbf{B}}+\tau_{2} \kappa^{2}(\vec{E} \cdot \overrightarrow{\mathbf{B}}) \overrightarrow{\mathbf{B}} \\
J_{5}^{t} & =\rho_{5}, \vec{J}_{5}=0,
\end{aligned}
$$

\footnotetext{
${ }^{4}$ We thank Dmitri Kharzeev for proposing us this study.

5 In principle it is not excluded that the charge densities $\rho, \rho_{5}$ could be spatially inhomogeneous. Yet such spatial inhomogeneity would render the derivative resummation highly complicated.
} 
where $\sigma_{e}$ is a $q^{2}=0$ limit of the electric conductivity introduced in (1), while $\tau_{1,2}$ are new TCFs. As with other TCFs, they are functionals of time derivative operator and become functions of frequency $\omega$ in Fourier space,

$\sigma_{e}\left[\partial_{t}\right] \longrightarrow \sigma_{e}(\omega), \tau_{1,2}\left[\partial_{t}\right] \longrightarrow \tau_{1,2}(\omega)$.

At the linear level (in external fields and hydro variables), the transport coefficient functions in $[9,68]$ were proved to be frame independent. Along this line of proof, we expect that $\sigma_{e}, \tau_{1,2}$ are also independent of the hydro frame choice. Imposing the continuity equation (2), the electric current is put on shell,

$$
\begin{aligned}
J^{i}=\sigma_{i j} E_{j}, \sigma_{i j}= & \underbrace{\sigma_{e}}_{\sigma_{\mathrm{T}}}\left(\delta_{i j}-\frac{\mathbf{B}_{i} \mathbf{B}_{j}}{\mathbf{B}^{2}}\right) \\
& +\underbrace{\left[\sigma_{e}-\left(\frac{12}{i \omega} \tau_{1}-\tau_{2}\right) \kappa^{2} \mathbf{B}^{2}\right]}_{\sigma_{\mathrm{L}}} \frac{\mathbf{B}_{i} \mathbf{B}_{j}}{\mathbf{B}^{2}},
\end{aligned}
$$

where the transverse conductivity $\sigma_{\mathrm{T}}$ is not affected by the magnetic field in contrast to the longitudinal conductivity $\sigma_{\mathrm{L}}$, which gets corrected by the magnetic field via the chiral anomaly. In Sect. 4.2 the TCFs $\tau_{1}, \tau_{2}$ will be first analytically evaluated in the hydro limit and then numerically for arbitrary frequency.

While there is some overlap between our results and the literature, differences between the present study and those of [73-76] must be clarified. Utilising the weak electric field approximation (10), [73] analytically evaluated the magnetic field dependence of the longitudinal conductivity $\sigma_{\mathrm{L}}$ in DC limit, while [74] calculated its $\omega$-dependence. Back-reaction effects on $\sigma_{\mathrm{L}}$ were considered in [76]. References [77] performed a similar study, focussing on time evolution of the induced vector current, given some specially chosen initial profile for the electric field. All the studies [73-76] focussed on a weak electric field, in which the axial current vanishes. Therefore, our nonlinear results and particularly the axial charge separation current (66) appears as new. As for the linearised setup (10), [73-76] imposed the continuity equation and replaced the axial charge density $\rho_{5}$ in favour of the external electric and magnetic fields, so the vector current there is on shell. This is in contrast to our off-shell formalism. As we argued in our previous publications [7,9-12], only offshell construction reveals transport properties of the system in full. Particularly, there are three independent TCFs $\left(\sigma_{e}\right.$ and $\left.\tau_{1,2}\right)$ in the constitutive relation (11), all of which we are able to determine separately, compared to only two independent conductivities in (13).

Another difference worth mentioning is that we explicitly trace all the effects in the induced current that arise from the relative angle between $\vec{E}(t)$ and $\overrightarrow{\mathbf{B}}$ fields. This is in contrast to $[74,77]$, which limited their study to the case of parallel fields only, primarily focussing on the longitudinal electric conductivity $\sigma_{\mathrm{L}}$. By varying the relative angle between $\vec{E}(t)$ and $\overrightarrow{\mathbf{B}}$ fields, one can separate the anomaly-induced effects (parametrised by $\tau_{1}$ and $\tau_{2}$ ) from the ones that are not related to the anomaly $\left(\sigma_{e}\right)$.

The paper is structured as follows. In Sect. 2 we present the holographic model and outline the strategy of deriving the boundary currents from solutions of the anomalous Maxwell equations in the bulk. Section 3 presents the first part of our study: CME/CSE with static but varying in space magnetic field. In Sect. 4, CME/CSE in the presence of constant magnetic and time-varying electric fields are analysed. This study is further split into two subsections. The exploration of nonlinear phenomena in the induced vector/axial currents is done in Sect. 4.1. In Sect. 4.2 we focus on the linearised regime (10) and calculate the dependence of AC conductivity on magnetic field. Section 5 presents the conclusions. Two appendices supplement computations of Sects. 3 and 4.

\section{The holographic model: $U(1)_{V} \times U(1)_{A}$}

The holographic model is the $U(1)_{V} \times U(1)_{A}$ theory in the Schwarzschild- $-\mathrm{AdS}_{5}$ case. The chiral anomaly of the boundary field theory is modelled via the gauge Chern-Simons terms in the bulk action,

$S=\int \mathrm{d}^{5} x \sqrt{-g} \mathcal{L}+S_{\text {c.t. }}$,

where

$$
\begin{aligned}
\mathcal{L}= & -\frac{1}{4}\left(F^{V}\right)_{M N}\left(F^{V}\right)^{M N}-\frac{1}{4}\left(F^{a}\right)_{M N}\left(F^{a}\right)^{M N}+\frac{\kappa \epsilon^{M N P Q R}}{2 \sqrt{-g}} \\
& \times\left[3 A_{M}\left(F^{V}\right)_{N P}\left(F^{V}\right)_{Q R}+A_{M}\left(F^{a}\right)_{N P}\left(F^{a}\right)_{Q R}\right],
\end{aligned}
$$

and the counter-term action $S_{\text {c.t. }}$ is

$$
\begin{aligned}
S_{\text {c.t. }}= & \frac{1}{4} \log r \int \mathrm{d}^{4} x \sqrt{-\gamma}\left[\left(F^{V}\right)_{\mu \nu}\left(F^{V}\right)^{\mu \nu}\right. \\
& \left.+\left(F^{a}\right)_{\mu \nu}\left(F^{a}\right)^{\mu \nu}\right] .
\end{aligned}
$$

The field strengths $\left(F^{V}\right)_{M N}$ and $\left(F^{a}\right)_{M N}$ are defined as

$\left(F^{V}\right)_{M N}=\partial_{M} V_{N}-\partial_{N} V_{M},\left(F^{a}\right)_{M N}=\partial_{M} A_{N}-\partial_{N} A_{M}$.

$\epsilon^{M N P Q R}$ is the Levi-Civita symbol with the convention $\epsilon^{r t x y z}=+1$, and the Levi-Civita tensor is $\epsilon^{M N P Q R} / \sqrt{-g}$. Our choice for (16) is based on minimal subtraction, that is, the counter-term does not make a finite contribution to the boundary currents.

In the ingoing Eddington-Finkelstein coordinates, the spacetime metric is

$$
\mathrm{d} s^{2}=g_{M N} \mathrm{~d} x^{M} \mathrm{~d} x^{N}=2 \mathrm{~d} t \mathrm{~d} r-r^{2} f(r) \mathrm{d} t^{2}+r^{2} \delta_{i j} \mathrm{~d} x^{i} \mathrm{~d} x^{j},
$$


where $f(r)=1-1 / r^{4}$, so that the Hawking temperature (identified as temperature of the boundary theory) is normalised to $\pi T=1$. On the constant $r$ hypersurface $\Sigma$, the induced metric $\gamma_{\mu \nu}$ is

$\left.\mathrm{d} s^{2}\right|_{\Sigma}=\gamma_{\mu \nu} \mathrm{d} x^{\mu} \mathrm{d} x^{\nu}=-r^{2} f(r) \mathrm{d} t^{2}+r^{2} \delta_{i j} \mathrm{~d} x^{i} \mathrm{~d} x^{j}$.

The equations of motion for $V$ and $A$ fields are as follows.

Dynamical equations : $\mathrm{EV}^{\mu}=\mathrm{EA}^{\mu}=0$,

Constraint equations : $\mathrm{EV}^{r}=\mathrm{EA}^{r}=0$,

where

$$
\begin{aligned}
\mathrm{EV}^{M} \equiv & \nabla_{N}\left(F^{V}\right)^{N M}+\frac{3 \kappa \epsilon^{M N P Q R}}{\sqrt{-g}}\left(F^{a}\right)_{N P}\left(F^{V}\right)_{Q R}, \\
\mathrm{EA}^{M} \equiv & \nabla_{N}\left(F^{a}\right)^{N M}+\frac{3 \kappa \epsilon^{M N P Q R}}{2 \sqrt{-g}} \\
& \times\left[\left(F^{V}\right)_{N P}\left(F^{V}\right)_{Q R}+\left(F^{a}\right)_{N P}\left(F^{a}\right)_{Q R}\right] .
\end{aligned}
$$

The boundary currents are defined as

$J^{\mu} \equiv \lim _{r \rightarrow \infty} \frac{\delta S}{\delta V_{\mu}}, J_{5}^{\mu} \equiv \lim _{r \rightarrow \infty} \frac{\delta S}{\delta A_{\mu}}$,

which, in terms of the bulk fields, are

$$
\begin{aligned}
J^{\mu}= & \lim _{r \rightarrow \infty} \sqrt{-\gamma} \\
& \times\left\{\left(F^{V}\right)^{\mu M} n_{M}+\frac{6 \kappa \epsilon^{M \mu N Q R}}{\sqrt{-g}} n_{M} A_{N}\left(F^{V}\right)_{Q R}\right. \\
& \left.\quad-\widetilde{\nabla}_{\nu}\left(F^{V}\right)^{v \mu} \log r\right\}, \\
J_{5}^{\mu}= & \lim _{r \rightarrow \infty} \sqrt{-\gamma} \\
\times & \left\{\left(F^{a}\right)^{\mu M} n_{M}+\frac{2 \kappa \epsilon^{M \mu N Q R}}{\sqrt{-g}} n_{M} A_{N}\left(F^{a}\right)_{Q R}\right. \\
& \left.-\widetilde{\nabla}_{\nu}\left(F^{a}\right)^{v \mu} \log r\right\},
\end{aligned}
$$

where $n_{M}$ is the outpointing unit normal vector on the slice $\Sigma$, and $\vec{\nabla}$ is compatible with the induced metric $\gamma_{\mu \nu}$.

The currents (24) are defined independently of the constraint equations (21). Throughout this work, the radial gauge $V_{r}=A_{r}=0$ will be assumed. Consequently, in order to completely determine the boundary currents (25) it is sufficient to solve the dynamical equations (20) for the bulk gauge fields $V_{\mu}, A_{\mu}$ only, leaving the constraints aside. The constraint equations (21) give rise to the continuity equations (2). In this way, the currents' constitutive relations to be derived below are off shell.

It is useful to reexpress the currents (25) in terms of the coefficients of the near-boundary asymptotic expansion of the bulk gauge fields. Near $r=\infty$,

$$
V_{\mu}=\mathcal{V}_{\mu}+\frac{V_{\mu}^{(1)}}{r}+\frac{V_{\mu}^{(2)}}{r^{2}}-\frac{2 V_{\mu}^{\mathrm{L}}}{r^{2}} \log r+\mathcal{O}\left(\frac{\log r}{r^{3}}\right),
$$

$A_{\mu}=\frac{A_{\mu}^{(2)}}{r^{2}}+\mathcal{O}\left(\frac{\log r}{r^{3}}\right)$,

where

$$
V_{\mu}^{(1)}=\mathcal{F}_{t \mu}^{V}, 4 V_{\mu}^{\mathrm{L}}=\partial^{\nu} \mathcal{F}_{\mu \nu}^{V} .
$$

In (26) the constant term for $A_{\mu}$ is set zero given that axial external fields are turned off in our present study. The holographic dictionary implies that $\mathcal{V}_{\mu}$ is a gauge potential of external electromagnetic fields $\mathbf{E}$ and $\vec{B}$,

$$
E_{i}=\mathcal{F}_{i t}^{V}=\partial_{i} \mathcal{V}_{t}-\partial_{t} \mathcal{V}_{i}, B_{i}=\frac{1}{2} \epsilon_{i j k} \mathcal{F}_{j k}^{V}=\epsilon_{i j k} \partial_{j} \mathcal{V}_{k}
$$

When obtaining (26) and (27), only the dynamical equations (20) were utilised. The the near-boundary data $V_{\mu}^{(2)}$ and $A_{\mu}^{(2)}$ have to be determined by completely solving (20) from the horizon to the boundary. The currents (25) become

$$
J^{\mu}=\eta^{\mu \nu}\left(2 V_{v}^{(2)}+2 V_{v}^{\mathrm{L}}+\eta^{\sigma t} \partial_{\sigma} \mathcal{F}_{t v}^{V}\right), J_{5}^{\mu}=\eta^{\mu \nu} 2 A_{v}^{(2)} .
$$

The remainder of this section is to meant to outline the strategy for deriving the constitutive relations for $J^{\mu}$ and $J_{5}^{\mu}$. To this end, consider finite vector/axial charge densities exposed to external electromagnetic fields. Holographically, the charge densities and external fields are encoded in asymptotic behaviours of the bulk gauge fields. In the bulk, we will solve the dynamical equations (20) assuming some charge densities and external fields, but without specifying them explicitly.

Following [9] we start with the most general static and homogeneous profiles for the bulk gauge fields which solve the dynamical equations (20),

$V_{\mu}=\mathcal{V}_{\mu}-\frac{\rho}{2 r^{2}} \delta_{\mu t}, \quad A_{\mu}=-\frac{\rho_{5}}{2 r^{2}} \delta_{\mu t}$,

where $\mathcal{V}_{\mu}, \rho, \rho_{5}$ are all constants for the moment. The regularity requirement at $r=1$ fixes one integration constant for each $V_{i}$ and $A_{i}$. As explained below (27), the constant in $A_{\mu}$ is set to zero. Through (29), the boundary currents are

$J^{t}=\rho, \quad J^{i}=0 ; \quad J_{5}^{t}=\rho_{5}, \quad J_{5}^{i}=0$.

Hence, $\rho$ and $\rho_{5}$ are identified as the vector/axial charge densities.

Next, following the idea of fluid/gravity correspondence [14], we promote $\mathcal{V}_{\mu}, \rho, \rho_{5}$ into arbitrary functions of the boundary coordinates,

$\mathcal{V}_{\mu} \rightarrow \mathcal{V}_{\mu}\left(x_{\alpha}\right), \quad \rho \rightarrow \rho\left(x_{\alpha}\right), \quad \rho_{5} \rightarrow \rho_{5}\left(x_{\alpha}\right)$.

Then (30) ceases to be a solution of the dynamical equations (20). To have them satisfied, suitable corrections in $V_{\mu}$ and $A_{\mu}$ have to be introduced:

$$
V_{\mu}\left(r, x_{\alpha}\right)=\mathcal{V}_{\mu}\left(x_{\alpha}\right)-\frac{\rho\left(x_{\alpha}\right)}{2 r^{2}} \delta_{\mu t}+\mathbb{V}_{\mu}\left(r, x_{\alpha}\right),
$$


$A_{\mu}\left(r, x_{\alpha}\right)=-\frac{\rho_{5}\left(x_{\alpha}\right)}{2 r^{2}} \delta_{\mu t}+\mathbb{A}_{\mu}\left(r, x_{\alpha}\right)$,

where $\mathbb{V}_{\mu}, \mathbb{A}_{\mu}$ will be determined from solving (20). Appropriate boundary conditions have to be specified. First, $\mathbb{V}_{\mu}$ and $\mathbb{A}_{\mu}$ have to be regular over the whole integration interval of $r \in[1, \infty]$. Second, at the conformal boundary $r=\infty$, we require

$\mathbb{V}_{\mu} \rightarrow 0, \quad \mathbb{A}_{\mu} \rightarrow 0 \quad$ as $r \rightarrow \infty$

which amounts to fixing the external gauge potentials to be $\mathcal{V}_{\mu}$ and zero (for the axial fields). Additional integration constants will be fixed by the Landau frame convention for the currents,

$J^{t}=\rho\left(x_{\alpha}\right), \quad J_{5}^{t}=\rho_{5}\left(x_{\alpha}\right)$.

The Landau frame choice can be identified as a residual gauge fixing for the bulk fields.

The vector/axial chemical potentials are defined as

$$
\begin{aligned}
& \mu=V_{t}(r=\infty)-V_{t}(r=1)=\frac{1}{2} \rho-\mathbb{V}_{t}(r=1), \\
& \mu_{5}=A_{t}(r=\infty)-A_{t}(r=1)=\frac{1}{2} \rho_{5}-\mathbb{A}_{t}(r=1) .
\end{aligned}
$$

Generically, $\mu, \mu_{5}$ are nonlinear functionals of densities and external fields.

In terms of $\mathbb{V}_{\mu}$ and $\mathbb{A}_{\mu}$, the dynamical equations (20) are

$$
\begin{aligned}
0= & r^{3} \partial_{r}^{2} \mathbb{V}_{t}+3 r^{2} \partial_{r} \mathbb{V}_{t}+r \partial_{r} \partial_{k} \mathbb{V}_{k} \\
& +12 \kappa \epsilon^{i j k}\left[\partial_{r} \mathbb{A}_{i}\left(\partial_{j} \mathcal{V}_{k}+\partial_{j} \mathbb{V}_{k}\right)+\partial_{r} \mathbb{V}_{i} \partial_{j} \mathbb{A}_{k}\right] \\
0= & \left(r^{5}-r\right) \partial_{r}^{2} \mathbb{V}_{i}+\left(3 r^{4}+1\right) \partial_{r} \mathbb{V}_{i}+2 r^{3} \partial_{r} \partial_{t} \\
& \times \mathbb{V}_{i}-r^{3} \partial_{r} \partial_{i} \mathbb{V}_{t}+r^{2}\left(\partial_{t} \mathbb{V}_{i}-\partial_{i} \mathbb{V}_{t}\right) \\
& +r\left(\partial^{2} \mathbb{V}_{i}-\partial_{i} \partial_{k} \mathbb{V}_{k}\right)-\frac{1}{2} \partial_{i} \rho+r^{2}\left(\partial_{t} \mathcal{V}_{i}-\partial_{i} \mathcal{V}_{t}\right) \\
& +r\left(\partial^{2} \mathcal{V}_{i}-\partial_{i} \partial_{k} \mathcal{V}_{k}\right) \\
& +12 \kappa r^{2} \epsilon^{i j k} \\
& \times\left(\frac{1}{r^{3}} \rho_{5} \partial_{j} \mathcal{V}_{k}+\frac{1}{r^{3}} \rho_{5} \partial_{j} \mathbb{V}_{k}+\partial_{r} \mathbb{A}_{t} \partial_{j} \mathcal{V}_{k}+\partial_{r} \mathbb{A}_{t} \partial_{j} \mathbb{V}_{k}\right) \\
& -12 \kappa r^{2} \epsilon^{i j k} \partial_{r} \mathbb{A}_{j} \\
& \times\left[\left(\partial_{t} \mathcal{V}_{k}-\partial_{k} \mathcal{V}_{t}\right)+\left(\partial_{t} \mathbb{V}_{k}-\partial_{k} \mathbb{V}_{t}\right)+\frac{1}{2 r^{2}} \partial_{k} \rho\right] \\
& -12 \kappa r^{2} \epsilon^{i j k}\left\{\partial_{r} \mathbb{V}_{j}\left[\left(\partial_{t} \mathbb{A}_{k}-\partial_{k} \mathbb{A}_{t}\right)+\frac{1}{2 r^{2}} \partial_{k} \rho_{5}\right]\right. \\
& \left.-\partial_{j} \mathbb{A}_{k}\left(\partial_{r} \mathbb{V}_{t}+\frac{1}{r^{3}} \rho\right)\right\}
\end{aligned}
$$

$$
\begin{aligned}
0= & r^{3} \partial_{r}^{2} \mathbb{A}_{t}+3 r^{2} \partial_{r} \mathbb{A}_{t}+r \partial_{r} \partial_{k} \mathbb{A}_{k}+12 \kappa \epsilon^{i j k} \\
& \times\left[\partial_{r} \mathbb{V}_{i}\left(\partial_{j} \mathcal{V}_{k}+\partial_{j} \mathbb{V}_{k}\right)+\partial_{r} \mathbb{A}_{i} \partial_{j} \mathbb{A}_{k}\right]
\end{aligned}
$$

$$
\begin{aligned}
0= & \left(r^{5}-r\right) \partial_{r}^{2} \mathbb{A}_{i}+\left(3 r^{4}+1\right) \partial_{r} \mathbb{A}_{i} \\
& +2 r^{3} \partial_{r} \partial_{t} \mathbb{A}_{i}-r^{3} \partial_{r} \partial_{i} \mathbb{A}_{t}+r^{2}\left(\partial_{t} \mathbb{A}_{i}-\partial_{i} \mathbb{A}_{t}\right) \\
& +r\left(\partial^{2} \mathbb{A}_{i}-\partial_{i} \partial_{k} \mathbb{A}_{k}\right)-\frac{1}{2} \partial_{i} \rho_{5} \\
& +12 \kappa r^{2} \epsilon^{i j k}\left(\partial_{j} \mathcal{V}_{k}+\partial_{j} \mathbb{V}_{k}\right)\left(\partial_{r} \mathbb{V}_{t}+\frac{1}{r^{3}} \rho\right) \\
& -12 \kappa r^{2} \epsilon^{i j k} \partial_{r} \mathbb{V}_{j} \\
& \times\left[\left(\partial_{t} \mathcal{V}_{k}-\partial_{k} \mathcal{V}_{t}\right)+\left(\partial_{t} \mathbb{V}_{k}-\partial_{k} \mathbb{V}_{t}\right)+\frac{1}{2 r^{2}} \partial_{k} \rho\right] \\
& -12 \kappa r^{2} \epsilon^{i j k} \\
& \times\left\{\partial_{r} \mathbb{A}_{j}\left[\left(\partial_{t} \mathbb{A}_{k}-\partial_{k} \mathbb{A}_{t}\right)+\frac{1}{2 r^{2}} \partial_{k} \rho_{5}\right]\right. \\
& \left.-\partial_{j} \mathbb{A}_{k}\left(\partial_{r} \mathbb{A}_{t}+\frac{1}{r^{3}} \rho_{5}\right)\right\} .
\end{aligned}
$$

In the following sections we will present solutions to the dynamical equations (37)-(40) under the two independent setups discussed in Sect. 1 .

\section{$3 \mathrm{CME} / \mathrm{CSE}$ with time-independent inhomogeneous magnetic field}

In this section we consider the case in which the magnetic field is the only external field that is turned on. The magnetic field is assumed to be varying in space, but it should be time independent to avoid creating an electric field. There is no restriction on the charge densities $\rho, \rho_{5}$. From the general results (26) and (27),

$$
\begin{aligned}
\mathbb{V}_{t}, \mathbb{A}_{t} \sim \mathcal{O}\left(\frac{\log r}{r^{3}}\right), \quad \mathbb{V}_{i} \sim \mathcal{O}\left(\frac{\log r}{r^{2}}\right), \\
\mathbb{A}_{i} \sim \mathcal{O}\left(\frac{1}{r^{2}}\right), \quad \text { as } \quad r \rightarrow \infty .
\end{aligned}
$$

In obtaining large $r$ estimates for $\mathbb{V}_{t}$ and $\mathbb{A}_{t}$, the frame convention (35) was used to fix the coefficients of $1 / r^{2}$ in the near-boundary expansion for $V_{t}, A_{t}$ (thus those of $\mathbb{V}_{t}$ and $\left.\mathbb{A}_{t}\right)$. The dynamical equations (37)-(40) get simplified,

$$
\begin{aligned}
0= & r^{3} \partial_{r}^{2} \mathbb{V}_{t}+3 r^{2} \partial_{r} \mathbb{V}_{t}+r \partial_{r} \partial_{k} \mathbb{V}_{k} \\
& +12 \kappa \epsilon^{i j k}\left[\partial_{r} \mathbb{A}_{i}\left(\partial_{j} \mathcal{V}_{k}+\partial_{j} \mathbb{V}_{k}\right)+\partial_{r} \mathbb{V}_{i} \partial_{j} \mathbb{A}_{k}\right], \\
0= & \left(r^{5}-r\right) \partial_{r}^{2} \mathbb{V}_{i}+\left(3 r^{4}+1\right) \partial_{r} \mathbb{V}_{i} \\
& +2 r^{3} \partial_{r} \partial_{t} \mathbb{V}_{i}-r^{3} \partial_{r} \partial_{i} \mathbb{V}_{t}+r^{2}\left(\partial_{t} \mathbb{V}_{i}-\partial_{i} \mathbb{V}_{t}\right) \\
& +r\left(\partial^{2} \mathbb{V}_{i}-\partial_{i} \partial_{k} \mathbb{V}_{k}\right)-\frac{1}{2} \partial_{i} \rho+r \partial_{k} \mathcal{F}_{k i}^{V} \\
& +12 \kappa r^{2} \epsilon^{i j k} \partial_{r}\left(\mathbb{A}_{t}-\frac{\rho_{5}}{2 r^{2}}\right)\left(\partial_{j} \mathcal{V}_{k}+\partial_{j} \mathbb{V}_{k}\right) \\
& -12 \kappa r^{2} \epsilon^{i j k} \\
& \times\left\{\partial_{r} \mathbb{V}_{j}\left[\left(\partial_{t} \mathbb{A}_{k}-\partial_{k} \mathbb{A}_{t}\right)+\frac{1}{2 r^{2}} \partial_{k} \rho_{5}\right]\right. \\
& \left.-\partial_{j} \mathbb{A}_{k} \partial_{r}\left(\mathbb{V}_{t}-\frac{\rho}{2 r^{2}}\right)\right\} \\
& -12 \kappa r^{2} \epsilon^{i j k} \partial_{r} \mathbb{A}_{j} \\
& \times\left[\left(\partial_{t} \mathbb{V}_{k}-\partial_{k} \mathbb{V}_{t}\right)+\frac{1}{2 r^{2}} \partial_{k} \rho\right],
\end{aligned}
$$




$$
\begin{aligned}
0= & r^{3} \partial_{r}^{2} \mathbb{A}_{t}+3 r^{2} \partial_{r} \mathbb{A}_{t}+r \partial_{r} \partial_{k} \mathbb{A}_{k}+12 \kappa \epsilon^{i j k} \\
& \times\left[\partial_{r} \mathbb{V}_{i}\left(\partial_{j} \mathcal{V}_{k}+\partial_{j} \mathbb{V}_{k}\right)+\partial_{r} \mathbb{A}_{i} \partial_{j} \mathbb{A}_{k}\right] \\
0= & \left(r^{5}-r\right) \partial_{r}^{2} \mathbb{A}_{i}+\left(3 r^{4}+1\right) \partial_{r} \mathbb{A}_{i} \\
& +2 r^{3} \partial_{r} \partial_{t} \mathbb{A}_{i}-r^{3} \partial_{r} \partial_{i} \mathbb{A}_{t}+r^{2}\left(\partial_{t} \mathbb{A}_{i}-\partial_{i} \mathbb{A}_{t}\right) \\
& +r\left(\partial^{2} \mathbb{A}_{i}-\partial_{i} \partial_{k} \mathbb{A}_{k}\right)-\frac{1}{2} \partial_{i} \rho_{5} \\
& +12 \kappa r^{2} \epsilon^{i j k}\left(\partial_{j} \mathcal{V}_{k}+\partial_{j} \mathbb{V}_{k}\right) \partial_{r}\left(\mathbb{V}_{t}-\frac{\rho}{2 r^{2}}\right) \\
& -12 \kappa r^{2} \epsilon^{i j k} \\
& \times\left\{\partial_{r} \mathbb{A}_{j}\left[\left(\partial_{t} \mathbb{A}_{k}-\partial_{k} \mathbb{A}_{t}\right)+\frac{1}{2 r^{2}} \partial_{k} \rho_{5}\right]\right. \\
& \left.-\partial_{j} \mathbb{A}_{k} \partial_{r}\left(\mathbb{A}_{t}+\frac{\rho_{5}}{2 r^{2}}\right)\right\} \\
& -12 \kappa r^{2} \epsilon^{i j k} \partial_{r} \mathbb{V}_{j} \\
& \times\left[\left(\partial_{t} \mathbb{V}_{k}-\partial_{k} \mathbb{V}_{t}\right)+\frac{1}{2 r^{2}} \partial_{k} \rho\right] .
\end{aligned}
$$

For generic profiles of $\rho, \rho_{5}$ and $\vec{B}(\vec{x})$, nonlinearity makes it difficult to solve (42)-(45). To explore the general structure of the vector/axial currents, we rewrite the dynamical equations (42)-(45) into integral forms. In this way, the nearboundary asymptotic expansion for $\mathbb{V}_{\mu}$ and $\mathbb{A}_{\mu}$ could be extracted from the integral forms of (42)-(45). For simplicity, we address the details in Appendix A. Substituting the near-boundary behaviour (94)-(97) into (29) produces the results (3) and (4). As mentioned below (3) and (4), $G_{i}, H_{i}$ are functionals of $\rho, \rho_{5}, \vec{B}$ and are presented in (98) and (99). The formal analysis establishes the structure of $J_{\mu} / J_{\mu}^{5}$, particularly the "non-renormalisation" of CME and its gradient corrections.

We proceed with the hydrodynamic gradient expansion for $J_{\mu} / J_{\mu}^{5}$. This requires us to perturbatively solve the dynamical equations (42)-(45) within the boundary derivative expansion (5),

$\partial_{\mu}=\left(\partial_{t}, \partial_{i}\right) \longrightarrow\left(\lambda \partial_{t}, \lambda \partial_{i}\right)$

The corrections $\mathbb{V}_{\mu}$ and $\mathbb{A}_{\mu}$ are expandable in $\lambda$,

$\mathbb{V}_{\mu}=\sum_{n=1}^{\infty} \lambda^{n} \mathbb{V}_{\mu}^{[n]}, \quad \mathbb{A}_{\mu}=\sum_{n=1}^{\infty} \lambda^{n} \mathbb{A}_{\mu}^{[n]}$.

At each order in $\lambda, \mathbb{V}_{\mu}^{[n]}$ and $\mathbb{A}^{[n]}$ form a system of ordinary differential equations in $r$-coordinate, which can be solved via direct integration over $r$. The results for $\mathbb{V}_{\mu}^{[n]}$ and $\mathbb{A}^{[n]}$ up to $n=2$ can be found in Appendix A; see (100)-(106).

Substituting the first order solutions (100)-(102) into (98) and (99) generates a hydrodynamic expansion for $G_{i}, H_{i}$ up to second order in the gradient expansion (throughout this work, the electromagnetic fields are thought of as of first order in derivative counting),

$$
\begin{aligned}
G_{i}(x=\infty)= & -\frac{\pi}{8} \partial_{t} \partial_{i} \rho \\
& +\left(\frac{3}{2} \pi+3 \log 2\right) \kappa \partial_{t} \rho_{5} B_{i} \\
& +18(1-2 \log 2) \kappa^{2}\left(\rho_{5}^{2}+\rho^{2}\right) \\
& \times \epsilon^{i j k} \partial_{j} B_{k}+18(2-3 \log 2) \kappa^{2} \epsilon^{i j k} \\
& \times\left(\rho_{5} \partial_{j} \rho_{5} B_{k}+\rho \partial_{j} \rho B_{k}\right)+\mathcal{O}\left(\partial^{3}\right), \\
H_{i}(x=\infty)= & -\frac{\pi}{8} \partial_{t} \partial_{i} \rho_{5} \\
& +\left(\frac{3}{2} \pi+3 \log 2\right) \kappa \partial_{t} \rho B_{i} \\
& +36(1-2 \log 2) \kappa^{2} \rho \rho_{5} \epsilon^{i j k} \partial_{j} B_{k} \\
& +18(2-3 \log 2) \kappa^{2} \epsilon^{i j k} \\
& \times\left(\rho_{5} \partial_{j} \rho B_{k}+\rho \partial_{j} \rho_{5} B_{k}\right)+\mathcal{O}\left(\partial^{3}\right) .
\end{aligned}
$$

Meanwhile, the second order results (103) and (104) give rise to the gradient expansion of chemical potentials (36)

$\mu=\frac{\rho}{2}+\frac{1}{16}(\pi-2 \log 2) \partial^{2} \rho-\frac{3}{4}(\pi-2 \log 2)$

$\kappa B_{k} \partial_{k} \rho_{5}+18(1-2 \log 2) \kappa^{2} \rho B^{2}+\mathcal{O}\left(\partial^{3}\right)$,

$\mu_{5}=\mu\left(\rho \leftrightarrow \rho_{5}\right)$.

In principle, the second order results (103)-(106) could be inserted into (98) and (99), producing derivative expansion for $G_{i}(x=\infty)$ and $H_{i}(x=\infty)$ up to third order. However, at third order $\mathcal{O}\left(\partial^{3}\right)$, computing $G_{i}, H_{i}$ becomes quite involved. Therefore, at third order $\mathcal{O}\left(\partial^{3}\right)$ we decided to track only terms linear in $\rho, \rho_{5}$. As a result, we are able to identify the first anomalous correction to the diffusion constant $\mathcal{D}_{0}$ due to magnetic field. The final expressions are

$$
\begin{aligned}
G_{i}^{[3]}(x=\infty)= & \frac{\pi^{2}}{48} \partial_{t}^{2} \partial_{i} \rho+\frac{1}{16}(\pi-2 \log 2) \partial^{2} \partial_{i} \rho \\
& +12 \#_{1} \kappa \partial_{t}^{2} \rho_{5} B_{i}-\frac{\pi^{2}}{8} \kappa \\
& \times\left[\partial^{2}\left(\rho_{5} B_{i}\right)-\partial_{i} \partial_{k}\left(\rho_{5} B_{k}\right)\right] \\
& +\frac{3}{4}(\pi-2 \log 2) \kappa \partial_{i}\left(B_{k} \partial_{k} \rho_{5}\right) \\
& +\frac{18(1-2 \log 2) \kappa^{2} B^{2} \partial_{i} \rho}{} \\
& +18(1-2 \log 2) \kappa^{2} \rho \partial_{i} B^{2} \\
& +\mathcal{O}\left(\rho^{2}, \rho_{5}^{2}, \rho \rho_{5}\right), \\
H_{i}^{[3]}(x=\infty)= & G_{i}^{[3]}(x=\infty)\left(\rho \leftrightarrow \rho_{5}\right),
\end{aligned}
$$

where $\#_{1}$ in (51) is given by the integral

$\#_{1} \equiv \frac{1}{2} \int_{1}^{\infty} \mathrm{d} y\left[2 y \partial_{y} b_{2}(y)+b_{2}(y)\right] \approx 0.362$,

where $b_{2}(r)$ is given in (109). The underlined term in (51) is a $\kappa^{2} B^{2}$-correction to the diffusion constant $\mathcal{D}_{0}$. Given that the lowest order anomalous correction to the diffusion constant is negative, it is interesting to explore this effect further for arbitrary magnitude of the magnetic field, which, however, goes beyond the scope of the present study. 
Our results for $J^{\mu}$ and $J_{5}^{\mu}$ can be used to explore dispersion relations for free modes propagating in the chiral medium. We consider a constant magnetic field only. Let us take a plane wave ansatz for the vector/axial charge densities $\rho=\delta \rho \exp (-i \omega t+\vec{q} \cdot \vec{x}), \rho_{5}=\delta \rho_{5} \exp (-i \omega t+\vec{q} \cdot \vec{x})$.

Then the continuity equation (2) becomes

$a \delta \rho+b \delta \rho_{5}=0, b \delta \rho+a \delta \rho_{5}=0$

which has a nontrivial solution when and only when

$a^{2}=b^{2} \Longrightarrow a= \pm b$,

where

$$
\begin{aligned}
a= & -i \omega+\frac{1}{2} q^{2}+9(\pi-2 \log 2) \kappa^{2}(\vec{q} \cdot \overrightarrow{\mathbf{B}})^{2} \\
& +216(1-2 \log 2) \kappa^{3} \mathbf{B}^{2} i \vec{q} \cdot \overrightarrow{\mathbf{B}}+\frac{\pi}{8} i \omega q^{2} \\
& -\frac{\pi^{2}}{48} \omega^{2} q^{2}-\frac{1}{16}(\pi-2 \log 2) q^{4} \\
& +18(1-2 \log 2) \kappa^{2} \mathbf{B}^{2} q^{2}, \\
b= & 6 \kappa i \vec{q} \cdot \overrightarrow{\mathbf{B}}-\frac{3}{4}(\pi-2 \log 2) \kappa q^{2} \vec{q} \cdot \overrightarrow{\mathbf{B}} \\
& -\left(\frac{3}{2} \pi+3 \log 2\right) \kappa \omega \vec{q} \cdot \overrightarrow{\mathbf{B}}+12 \#_{1} \kappa \omega^{2} i \vec{q} \cdot \overrightarrow{\mathbf{B}} \\
& +\frac{3}{4}(\pi-2 \log 2) \kappa q^{2} \vec{q} \cdot \overrightarrow{\mathbf{B}} .
\end{aligned}
$$

Solving (56) leads to the B-corrected dispersion relation, as summarised in (7).

\section{$4 \mathrm{CME} / \mathrm{CSE}$ with constant magnetic and time-dependent electric fields}

Creating systems with chiral imbalance $\left(\mu_{5} \neq 0\right)$ experimentally is problematic. In this section we consider a special setup in which the axial chemical potential $\mu_{5}$ is not imposed externally but rather is induced dynamically through the chiral anomaly. This setup is of particular interest due to intriguing possibility for it to be realised experimentally in chiral condensed matter systems. Consider a constant magnetic field $\overrightarrow{\mathbf{B}}$ and a time-dependent homogeneous electric field $\vec{E}(t)$. We also assume the charge densities to be spatially homogeneous as well. ${ }^{6}$ The continuity equation (2) degenerates to

$\partial_{t} J^{t}=0, \partial_{t} J_{5}^{t}=12 \kappa \vec{E} \cdot \overrightarrow{\mathbf{B}}$,

which implies that the vector charge density is constant while the axial charge density has nontrivial time dependence inher-

\footnotetext{
6 While from the continuity equation (2) the charge densities can still have a nontrivial spatial-dependence, we found that such spatial inhomogeneity of the charge densities would make the gradient resummation out of control.
}

ited from $\mathbf{E}(t)$. The setup under consideration is

$\rho=0, \rho_{5}=\rho_{5}(t), \vec{E}=\vec{E}(t), \overrightarrow{\mathbf{B}}=$ constant.

Under the frame convention (35), the corrections $\mathbb{V}_{\mu}$ and $\mathbb{A}_{\mu}$ of (33) depend on $r$ and $t$ only. As a result, the dynamical equations (37)-(40) are reduced to

$$
\begin{aligned}
0= & r^{3} \partial_{r}^{2} \mathbb{V}_{t}+3 r^{2} \partial_{r} \mathbb{V}_{t}+12 \kappa \partial_{r} \mathbb{A}_{k} \mathbf{B}_{k}, \\
0= & \left(r^{5}-r\right) \partial_{r}^{2} \mathbb{V}_{i}+\left(3 r^{4}+1\right) \partial_{r} \mathbb{V}_{i}+2 r^{3} \partial_{r} \partial_{t} \mathbb{V}_{i} \\
& +r^{2} \partial_{t} \mathbb{V}_{i}-r^{2} E_{i}+12 \kappa r^{2} \partial_{r} \mathbb{A}_{t} \mathbf{B}_{i} \\
& +\frac{12}{r} \kappa \rho_{5} \mathbf{B}_{i}-12 \kappa r^{2} \epsilon^{i j k} \partial_{r} \mathbb{A}_{j}\left(\partial_{t} \mathbb{V}_{k}-E_{k}\right) \\
& -12 \kappa r^{2} \epsilon^{i j k} \partial_{r} \mathbb{V}_{j} \partial_{t} \mathbb{A}_{k}, \\
0= & r^{3} \partial_{r}^{2} \mathbb{A}_{t}+3 r^{2} \partial_{r} \mathbb{A}_{t}+12 \kappa \partial_{r} \mathbb{V}_{k} \mathbf{B}_{k}, \\
0= & \left(r^{5}-r\right) \partial_{r}^{2} \mathbb{A}_{i}+\left(3 r^{4}+1\right) \partial_{r} \mathbb{A}_{i}+2 r^{3} \partial_{r} \partial_{t} \mathbb{A}_{i} \\
& +r^{2} \partial_{t} \mathbb{A}_{i}+12 \kappa r^{2} \partial_{r} \mathbb{V}_{t} \mathbf{B}_{i} \\
& -12 \kappa r^{2} \epsilon^{i j k} \partial_{r} \mathbb{V}_{j}\left(\partial_{t} \mathbb{V}_{k}-E_{k}\right) \\
& -12 \kappa r^{2} \epsilon^{i j k} \partial_{r} \mathbb{A}_{j} \partial_{t} \mathbb{A}_{k} .
\end{aligned}
$$

4.1 Nonlinear phenomena: general analysis and derivative expansion

The objective of this subsection is to show that beyond linearised limit (69) the setup (60) also induces a non-vanishing axial current $\vec{J}_{5}$, which has been omitted in the literature. To this end, as in Sect. 3, we first give a fully nonlinear analysis for the dynamical equations (61)-(64), followed by perturbative calculations for $\mathbb{V}_{\mu}, \mathbb{A}_{\mu}$ within the derivative expansion (5). All calculational details are addressed in Appendix B.

As in Sect. 3 the formal analysis are based on rewriting the dynamical equations (61)-(64) into integral form, from which one could deduce the near-boundary asymptotic behaviours for $\mathbb{V}_{\mu}, \mathbb{A}_{\mu}$. The results can be found in (112)(115). Plugged them into (29), the near-boundary behaviour for $\mathbb{V}_{\mu}, \mathbb{A}_{\mu}$ presented in (112)-(115) is translated into boundary currents (8) and (9). Generically, the quantities $\mathbb{V}_{i}(1)$, $\mathbb{A}_{i}(1), \bar{G}_{i}(x=\infty)$ and $\bar{H}_{i}(x=\infty)$ in (8) and (9) cannot be computed analytically. However, as in Sect. 3 , the formal analysis determines the generic forms for $J^{\mu} / J_{5}^{\mu}$.

Within the gradient expansion (5), we perturbatively solve the dynamical equations (61)-(64). Up to second order $\mathcal{O}\left(\partial^{2}\right), \mathbb{V}_{\mu}, \mathbb{A}_{\mu}$ are shown in (118)-(121). The perturbative solutions (118)-(121) can be plugged into (116) and (117) to generate hydrodynamic expansion for $J^{\mu} / J_{5}^{\mu}$ :

$$
\begin{aligned}
\vec{J}= & 12 \kappa \mu_{5} \overrightarrow{\mathbf{B}}+\vec{E}-\frac{\log 2}{2} \partial_{t} \vec{E}-\frac{\pi^{2}}{24} \partial_{t}^{2} \vec{E} \\
& -\left(\frac{3}{2} \pi+3 \log 2\right) \kappa \partial_{t} \rho_{5} \overrightarrow{\mathbf{B}} \\
& +9 \pi^{2} \kappa^{3} \rho_{5}(\overrightarrow{\mathbf{B}} \times \vec{E}) \times \vec{E}+12 \#_{1} \kappa \partial_{t}^{2} \rho_{5} \overrightarrow{\mathbf{B}}+\mathcal{O}\left(\partial^{4}\right),
\end{aligned}
$$




$$
\begin{aligned}
\vec{J}_{5}= & 12 \kappa \mu \overrightarrow{\mathbf{B}}-36 \log 2 \kappa^{2} \rho_{5} \overrightarrow{\mathbf{B}} \times \vec{E} \\
& +\frac{3}{2}\left(\pi^{2}+3 \pi \log 2+6 \log ^{2} 2\right) \kappa^{2} \partial_{t} \rho_{5} \overrightarrow{\mathbf{B}} \times \vec{E} \\
& -\frac{3}{8}\left(48 \mathcal{C}+\pi^{2}-12 \pi \log 2\right) \kappa^{2} \rho_{5} \overrightarrow{\mathbf{B}} \times \partial_{t} \vec{E}+\mathcal{O}\left(\partial^{4}\right),
\end{aligned}
$$

where $\mathcal{C}$ is a Catalan constant and $\#_{1}$ is known numerically only,

$\#_{1} \approx 0.362$.

Up to second order in derivatives $\mathcal{O}\left(\partial^{2}\right)$, the chemical potentials (36) are $^{7}$

$$
\begin{aligned}
\mu= & +\mathcal{O}\left(\partial^{3}\right), \quad \mu_{5}=\frac{1}{2} \rho_{5}+\frac{3}{2}(\pi-2 \log 2) \kappa \vec{E} \cdot \overrightarrow{\mathbf{B}} \\
& +18(1-2 \log 2) \kappa^{2} \rho_{5} \mathbf{B}^{2}+\mathcal{O}\left(\partial^{3}\right) .
\end{aligned}
$$

Evaluated on shell via (2), the axial current $J_{5}^{i}$ is fully nonlinear in the amplitude of the electric field $\vec{E}(t)$, as clear from (66).

\subsection{Linear in $\vec{E}$ phenomena}

In the previous subsection we focussed on hydrodynamic regime, in which we were able to identify some nonlinear phenomena. Below, we proceed with an alternative approximation, that is, the weak electric field approximation (10):

$\rho_{5}(t) \sim \mathcal{O}(\epsilon), \quad \vec{E}(t) \sim \mathcal{O}(\epsilon), \quad \overrightarrow{\mathbf{B}} \sim \mathcal{O}\left(\epsilon^{0}\right)$.

The scaling of $\rho_{5}$ follows from the continuity equation (59). Both corrections $\mathbb{V}_{\mu}$ and $\mathbb{A}_{\mu}$ are of order $\mathcal{O}(\epsilon)$ too. The dynamical equations (61)-(64) get further simplified,

$0=r^{3} \partial_{r}^{2} \mathbb{V}_{t}+3 r^{2} \partial_{r} \mathbb{V}_{t}+12 \kappa \partial_{r} \mathbb{A}_{k} \mathbf{B}_{k}$,

$0=\left(r^{5}-r\right) \partial_{r}^{2} \mathbb{V}_{i}+\left(3 r^{4}+1\right) \partial_{r} \mathbb{V}_{i}+2 r^{3} \partial_{r} \partial_{t} \mathbb{V}_{i}$

$+r^{2} \partial_{t} \mathbb{V}_{i}-r^{2} E_{i}+12 \kappa r^{2}\left(\partial_{r} \mathbb{A}_{t}+\frac{\rho_{5}}{r^{3}}\right) \mathbf{B}_{i}$,

$0=r^{3} \partial_{r}^{2} \mathbb{A}_{t}+3 r^{2} \partial_{r} \mathbb{A}_{t}+12 \kappa \partial_{r} \mathbb{V}_{k} \mathbf{B}_{k}$

$0=\left(r^{5}-r\right) \partial_{r}^{2} \mathbb{A}_{i}+\left(3 r^{4}+1\right) \partial_{r} \mathbb{A}_{i}+2 r^{3} \partial_{r} \partial_{t} \mathbb{A}_{i}$

$$
+r^{2} \partial_{t} \mathbb{A}_{i}+12 \kappa r^{2} \partial_{r} \mathbb{V}_{t} \mathbf{B}_{i} .
$$

Integrating (70) and (72) over $r$ once, we get

$\partial_{r} \mathbb{V}_{t}=-\frac{12 \kappa}{r^{3}} \mathbb{A}_{k} \mathbf{B}_{k}, \partial_{r} \mathbb{A}_{t}=-\frac{12 \kappa}{r^{3}} \mathbb{V}_{k} \mathbf{B}_{k}$,

where the frame convention (35) was used to fix the integration constant. Equation (74) makes it possible to decouple $\mathbb{V}_{i}, \mathbb{A}_{i}$ from $\mathbb{V}_{t}, \mathbb{A}_{t}$. Consequently, (71) and (73) become

\footnotetext{
7 While we suspect that the chemical potential $\mu$ is zero to all orders in the gradient expansion, we have not been able to prove this.
}

$$
\begin{aligned}
0= & \left(r^{5}-r\right) \partial_{r}^{2} \mathbb{V}_{i}+\left(3 r^{4}+1\right) \partial_{r} \mathbb{V}_{i}+2 r^{3} \partial_{r} \partial_{t} \mathbb{V}_{i} \\
& +r^{2} \partial_{t} \mathbb{V}_{i}-r^{2} E_{i}+\frac{12 \kappa}{r} \mathbf{B}_{i}\left(\rho_{5}-12 \kappa \mathbb{V}_{k} \mathbf{B}_{k}\right), \\
0= & \left(r^{5}-r\right) \partial_{r}^{2} \mathbb{A}_{i}+\left(3 r^{4}+1\right) \partial_{r} \mathbb{A}_{i}+2 r^{3} \partial_{r} \partial_{t} \mathbb{A}_{i} \\
& +r^{2} \partial_{t} \mathbb{A}_{i}-\frac{144}{r} \kappa^{2} \mathbf{B}_{i}\left(\mathbb{A}_{k} \mathbf{B}_{k}\right) .
\end{aligned}
$$

The homogeneity property of (76), combined with the regularity requirement at $r=1$ and vanishing boundary condition at $r=\infty$ for $\mathbb{A}_{i}$, fixes $\mathbb{A}_{i}=0$ completely. From (74), $\mathbb{V}_{t}=0$. That is,

$\mathbb{V}_{t}=\mathbb{A}_{i}=0$.

Therefore, at order $\mathcal{O}(\epsilon)$, the axial current $\vec{J}_{5}=0$ as read off from (29). This is in contrast with the nonlinear analysis of Sect. 4.1.

The differential equation (76) is linear in the correction $\mathbb{V}_{i}$. Therefore, (76) can be solved via the technique developed in [7,10-12]. The bulk equations reduce to linear inhomogeneous partial differential equations while the inhomogeneous terms are built from boundary derivatives of the fluiddynamic variables and external fields. The equations then can be exactly solved using the Green function formalism: the bulk fields are decomposed in terms of all possible basic vector structures constructed from the fluid-dynamic variables and external fields. These decomposition coefficients (components of the inverse Green function) are functions of the holographic radial coordinate and functionals of the boundary derivative operators. The functional dependence of the decomposition coefficients on the boundary derivative operation encodes all-order linear derivatives in the constitutive relations. Transformed into momentum space, the bulk equations give rise to ordinary differential equations for those decomposition coefficients, which are RG-like equations in AdS space. Solving the RG-like equations completely determines the fluid's constitutive relations and all transport coefficients. Below we implement these steps.

$\mathbb{V}_{i}$ is decomposed as ${ }^{8}$

$\mathbb{V}_{i}=C_{1} E_{i}+C_{2} \kappa \rho_{5} \mathbf{B}_{i}+C_{3} \kappa^{2}(\vec{E} \cdot \overrightarrow{\mathbf{B}}) \mathbf{B}_{i}$,

where

$C_{i}=C_{i}\left(r, \partial_{t}\right) \rightarrow C_{i}(r, \omega), \quad i=1,2,3$.

The decomposition coefficients $C_{i}$ satisfy the partially decoupled ordinary differential equations (ODEs),

$$
\begin{aligned}
0= & \left(r^{5}-r\right) \partial_{r}^{2} C_{1}+\left(3 r^{4}+1\right) \partial_{r} C_{1}-2 i \omega r^{3} \partial_{\mathrm{r}} C_{1} \\
& -i \omega r^{2} C_{1}-r^{2},
\end{aligned}
$$

${ }^{8}$ In the decomposition for $\mathbb{V}_{i}$, one could have included a term $C_{4} \vec{E} \times \overrightarrow{\mathbf{B}}$. However, the coefficient $C_{4}$ would satisfy a homogeneous ODE. Under the same arguments leading to $\mathbb{A}_{i}=0, C_{4}$ has to be zero too. 
$0=\left(r^{5}-r\right) \partial_{\mathrm{r}}^{2} C_{2}+\left(3 r^{4}+1\right) \partial_{\mathrm{r}} C_{2}-2 i \omega r^{3} \partial_{\mathrm{r}} C_{2}-i \omega r^{2} C_{2}$ $+\frac{12}{r}\left(1-12 \kappa^{2} \mathbf{B}^{2} C_{2}\right)$,

$$
\begin{aligned}
0= & \left(r^{5}-r\right) \partial_{\mathrm{r}}^{2} C_{3}+\left(3 r^{4}+1\right) \partial_{\mathrm{r}} C_{3}-2 i \omega r^{3} \partial_{\mathrm{r}} C_{3} \\
& -i \omega r^{2} C_{3}-\frac{144}{r}\left(C_{1}+\kappa^{2} \mathbf{B}^{2} C_{3}\right) .
\end{aligned}
$$

While $C_{1}$ does not feel the effect of the magnetic field, $C_{2,3}$ have a nontrivial dependence on the magnetic field via $\kappa^{2} \mathbf{B}^{2}$.

Near $r=\infty$, the pre-asymptotic expansions of the $C_{i}$ are

$C_{1} \rightarrow-\frac{1}{r}+\frac{c_{1}}{r^{2}}-\frac{i \omega \log r}{2 r^{2}}+\mathcal{O}\left(\frac{\log r}{r^{3}}\right)$,

$C_{2} \rightarrow \frac{c_{2}}{r^{2}}+\mathcal{O}\left(\frac{1}{r^{3}}\right), \quad C_{3} \rightarrow \frac{c_{3}}{r^{2}}+\mathcal{O}\left(\frac{1}{r^{3}}\right)$,

where the $c_{i}$ are boundary data and have to be fixed through full solution of (80)-(82) from the horizon $r=1$ to the conformal boundary $r=\infty$. From (29), the conductivities of (11) are determined by the boundary data $c_{i}$,

$\sigma_{e}=2 c_{1}-\frac{1}{2} i \omega, \quad \tau_{1}=2 c_{2}, \quad \tau_{2}=2 c_{3}$.

The ODE for $C_{1}$ was solved in [9]. The conductivity $\sigma_{e}$, which is computed from $C_{1}$, was completely determined and explored in [9], while only $q=0$ limit enters into our current study (the results are quoted below). We therefore focus on the remaining two conductivities $\tau_{1}, \tau_{2}$, both induced by the chiral anomaly. As is obvious from (80)-(82), $\tau_{1}, \tau_{2}$ depend on the magnetic field via $\kappa^{2} \mathbf{B}^{2}$.

Using the continuity equation (59), the constitutive relations (11) are put into a linear response form, from which on-shell current-current correlators can be read off. Since the electric field is the only external perturbation that is turned on, it is possible to compute only a subset of all two-point correlators in the theory,

$$
\begin{aligned}
\left\langle J^{i} J^{j}\right\rangle= & \underbrace{i \omega \sigma_{e}}_{G^{\mathrm{T}}}\left(\delta_{i j}-\frac{\mathbf{B}_{i} \mathbf{B}_{j}}{\mathbf{B}^{2}}\right) \\
& +\underbrace{\left[i \omega \sigma_{e}-\left(12 \tau_{1}-i \omega \tau_{2}\right) \kappa^{2} \mathbf{B}^{2}\right]}_{G^{\mathrm{L}}} \frac{\mathbf{B}_{i} \mathbf{B}_{j}}{\mathbf{B}^{2}}, \\
\left\langle J_{5}^{t} J^{i}\right\rangle= & -12 \kappa \mathbf{B}_{i}, \\
\left\langle J^{t} J^{i}\right\rangle= & \left\langle J_{5}^{i} J^{j}\right\rangle=0,
\end{aligned}
$$

where $\left\langle J^{i} J^{j}\right\rangle$ is split into transverse $\left(G^{\mathrm{T}}\right)$ and longitudinal $\left(G^{\mathrm{L}}\right)$ components with respect to the direction of $\overrightarrow{\mathbf{B}}$. To determine the remaining current-current correlators we would have to introduce additional field perturbations, particularly an axial external field, which is beyond the scope of this paper.
To evaluate the TCFs $\tau_{1}, \tau_{2}$, we have to completely solve the ODEs (80)-(82). We first analytically solve them when $\omega=0$. As a result, the DC limits $\tau_{1}^{0}$ (for arbitrary $\mathbf{B}$ ) and $\tau_{2}^{0}$ (up to leading $\mathbf{B}^{2}$-correction) are known analytically,

$$
\begin{aligned}
\tau_{1}^{0}= & \frac{\Gamma\left[3 / 4-\sqrt{1-144 \kappa^{2} \mathbf{B}^{2}} / 4\right] \Gamma\left[3 / 4+\sqrt{1-144 \kappa^{2} \mathbf{B}^{2}} / 4\right]}{3 \kappa^{2} \mathbf{B}^{2} \Gamma\left[1 / 4-\sqrt{1-144 \kappa^{2} \mathbf{B}^{2}} / 4\right] \Gamma\left[1 / 4+\sqrt{1-144 \kappa^{2} \mathbf{B}^{2}} / 4\right]} \\
& \longrightarrow 6+216(1-2 \log 2) \kappa^{2} \mathbf{B}^{2}+\mathcal{O}\left(\mathbf{B}^{4}\right), \text { as } \mathbf{B} \rightarrow 0, \\
\tau_{2}^{0}= & 18(\pi-2 \log 2)+\#_{2} \kappa^{2} \mathbf{B}^{2}+\mathcal{O}\left(\mathbf{B}^{4}\right), \text { as } \mathbf{B} \rightarrow 0,
\end{aligned}
$$

where $\Gamma[z]$ is the Gamma function, and $\#_{2}$ is known numerically only,

$$
\begin{aligned}
\#_{2} \equiv & \int_{1}^{\infty} \frac{d r}{r^{3}}\left\{\int_{\mathrm{r}}^{\infty} \frac{72^{2} x \mathrm{~d} x}{x^{4}-1} \int_{1}^{x} \frac{\mathrm{d} y}{y}\right. \\
& \left.\times\left[\log \frac{(1+y)^{2}}{1+y^{2}}-2 \arctan (y)+\pi\right]\right\} \approx-495.268
\end{aligned}
$$

When the magnetic field is very strong, $\tau_{1}^{0}$ and $\tau_{2}^{0}$ behave similarly

$\tau_{1}^{0}, \tau_{2}^{0} \longrightarrow \frac{1}{\kappa \mathbf{B}}, \operatorname{as} \kappa \mathbf{B} \rightarrow \infty$.

The result for $\tau_{1}^{0}$ is in agreement with $[74,77]$. In the DC limit $\omega \rightarrow 0$, when the magnetic field is very strong, the on-shell vector current (13) behaves as

$J^{i} \rightarrow-12 \kappa \mathbf{B} \mathcal{V}_{i}$,

which is in agreement with [77]. When $\omega \rightarrow 0$ (DC limit), the current-current correlator is dominated by the chiral anomaly-induced effects $\sim \tau_{1}^{0}$. The DC limit is of interest for experiments with electric fields turned on adiabatically, such as the ones considered in [77]. Meanwhile, when $\omega \rightarrow 0$, the longitudinal conductivity $\sigma_{\mathrm{L}}$ in (13) is parametrised as

$\sigma_{\mathrm{L}}^{0}=\frac{i}{\omega} 12 \kappa^{2} \mathbf{B}^{2} \tau_{1}^{0}+\left[\sigma_{e}^{0}+\kappa^{2} \mathbf{B}^{2}\left(\tau_{2}^{0}-12 \tau_{1}^{1}\right)\right]$,

where $\sigma_{e}^{0}=1, \tau_{1}^{1}$ is the coefficient of $i \omega$ in the hydrodynamic expansion of $\tau_{1}$. For illustration, in Fig. 1 we show the $\kappa \mathbf{B}$ dependence of $\tau_{1}^{0}, \tau_{2}^{0}$ (divided by 5 to match scales), $\tau_{1}^{1}$ and $\operatorname{Re}\left(\sigma_{\mathrm{L}}^{0}\right)$. The behaviour of $\operatorname{Re}\left(\sigma_{\mathrm{L}}^{0}\right)$ agrees perfectly with that of [74].

In our calculation, $\operatorname{Re}\left(\sigma_{\mathrm{L}}^{0}\right)$ acquires a negative correction due to the magnetic field and eventually vanishes when the magnetic field gets large; see Fig. 1. This is in contrast with many related studies of negative magnetoresistivity, the phenomenon of enhancement of the longitudinal DC conductivity due to the magnetic field [78-83]. However, taking a strict DC limit in $\sigma_{\mathrm{L}}^{0}$ is problematic due to the explicit $1 / \omega$ divergence. The latter is frequently regularised by introduction of axial charge dissipation effects via shifting the frequency 

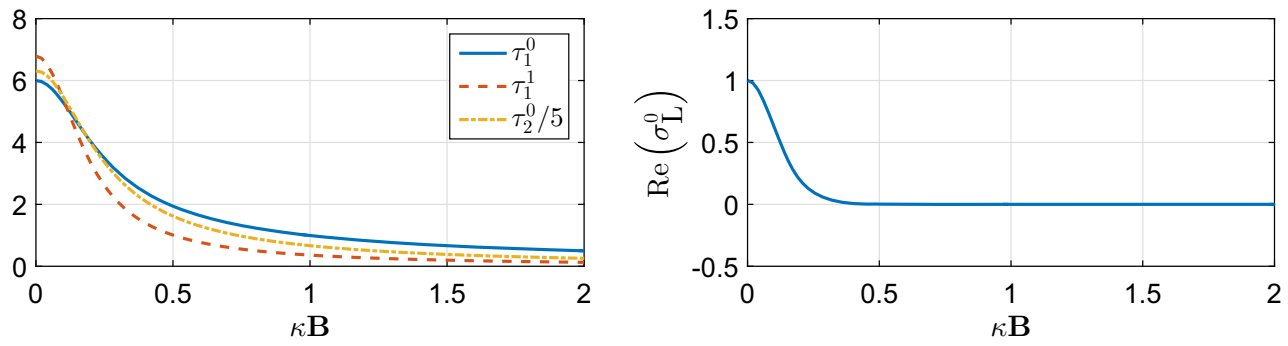

Fig. 1 DC conductivities $\tau_{1}^{0}, \tau_{2}^{0} / 5, \tau_{1}^{1}$, and $\operatorname{Re}\left(\sigma_{\mathrm{L}}^{0}\right)$ as a function of $\kappa \mathbf{B}$
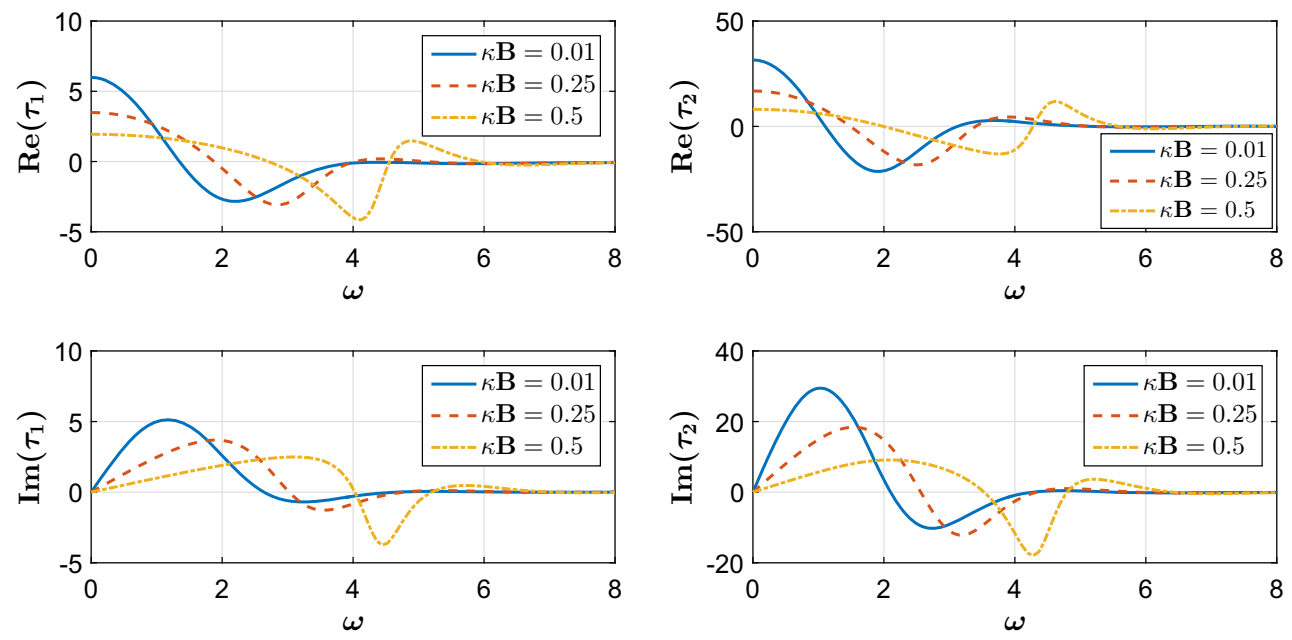

Fig. 2 AC conductivities $\tau_{1}$ and $\tau_{2}$ for different values of $\kappa \mathbf{B}$
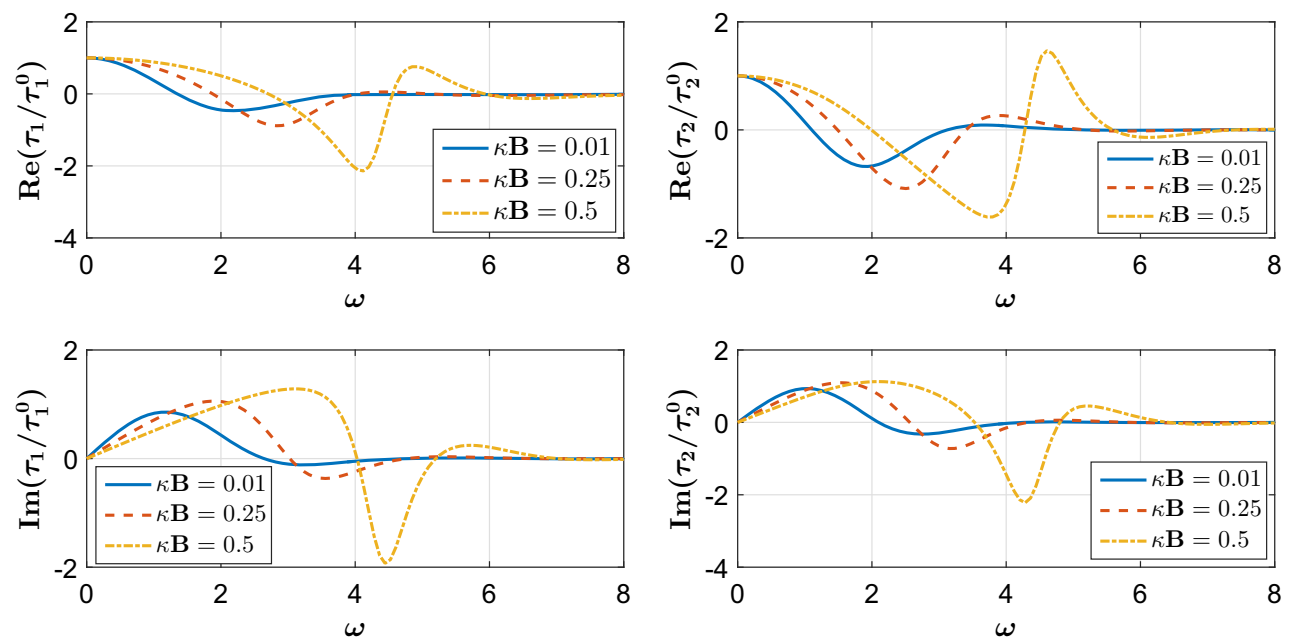

Fig. 3 Normalised AC conductivities $\tau_{1} / \tau_{1}^{0}$ and $\tau_{2} / \tau_{2}^{0}$ for different values of $\kappa \mathbf{B}$

$\omega \rightarrow \omega+i / \tau_{5}$, where $\tau_{5}$ corresponds to some relaxation time. The physics of this axial charge relaxation is beyond the scope of the present work. It was addressed within the holographic approach in [73-76]. These studies primarily rely on the Kubo formula.

For arbitrary $\omega$, we resort to numerical methods and solve ODEs (80)-(82) for representative values of $\kappa \mathbf{B}$. The numeri- cal procedure is identical to that of [68] and for all the numerical details we refer the reader to this publication. In Fig. 2 we show the $\omega$-dependence for $\tau_{1}$ and $\tau_{2}$ for sample choices of $\kappa \mathbf{B}$. In Fig. 3 we plot the normalised TCFs $\tau_{1} / \tau_{1}^{0}$ and $\tau_{2} / \tau_{2}^{0}$. Overall, $\tau_{1}$ and $\tau_{2}$ display quite similar dependences on the frequency $\omega$. After some oscillations, both $\tau_{1}$ and $\tau_{2}$ approach zero asymptotically. 

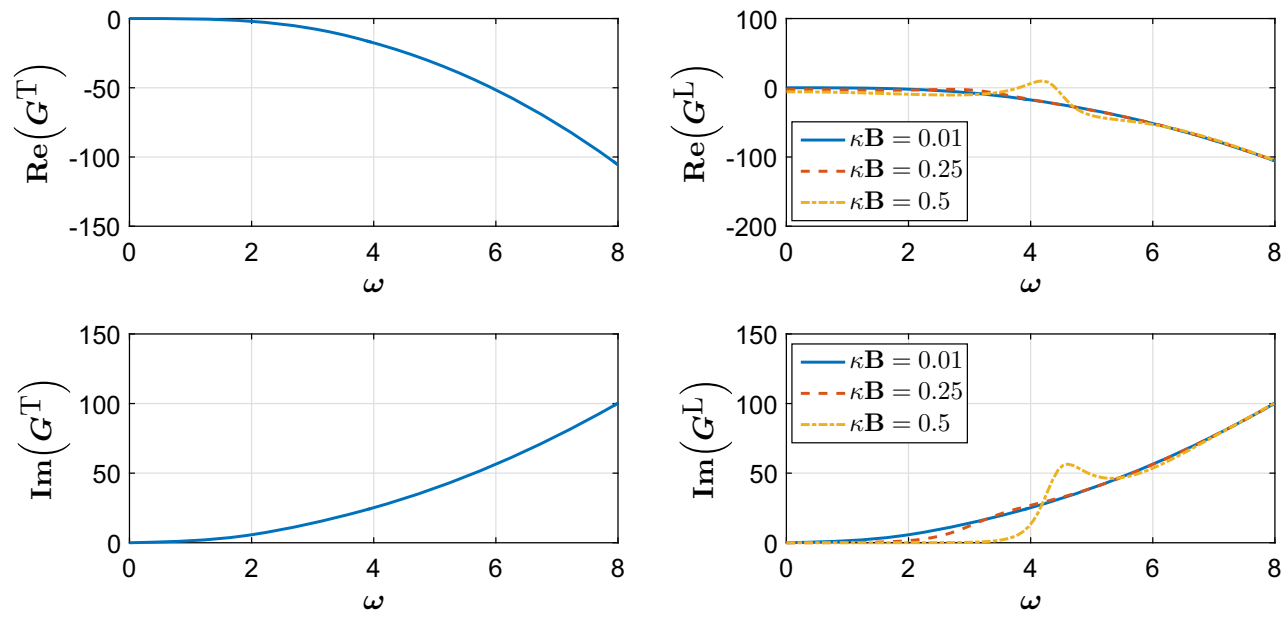

Fig. 4 Current-current correlators $G^{\mathrm{T}}$ (left) and $G^{\mathrm{L}}$ (right)
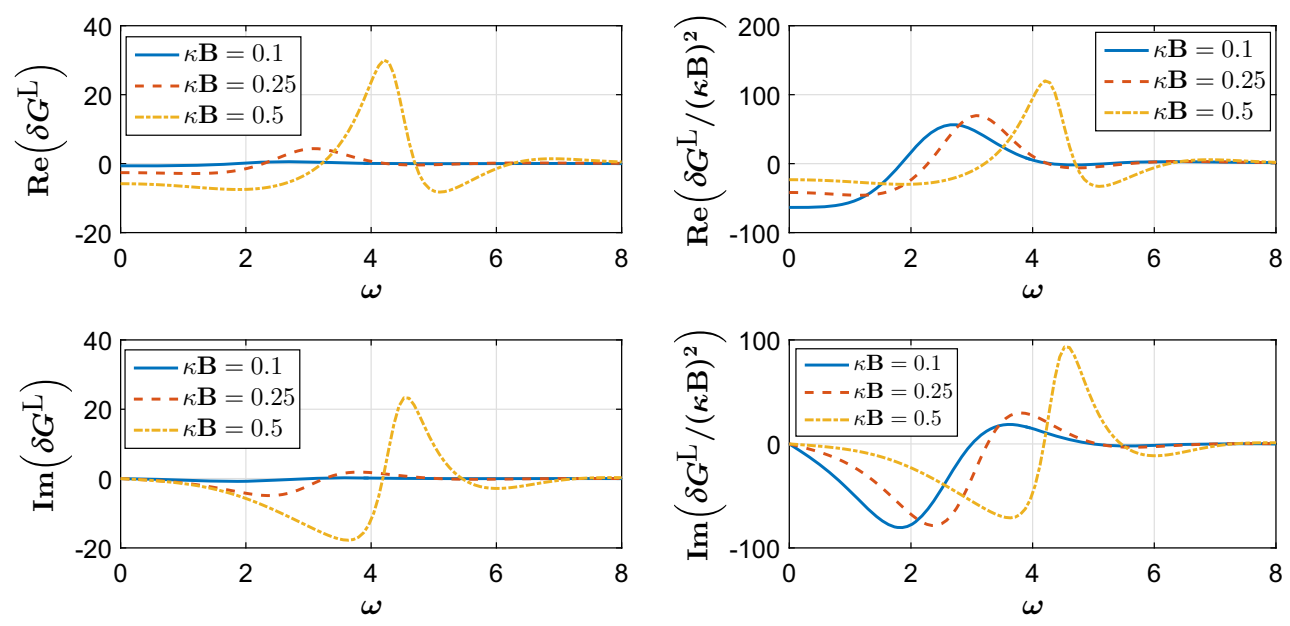

Fig. 5 Anomalous correction to correlator: $\delta G^{\mathrm{L}}($ left $)$ and $\delta G^{\mathrm{L}} /(\kappa \mathbf{B})^{2}($ right $)$

Approach to the asymptotic regime, however, depends on strength of the magnetic field. When $\kappa \mathbf{B}$ is increased, the asymptotic behaviour is delayed towards larger $\omega$. What is more intriguing is that increasing $\kappa \mathbf{B}$ renders $\tau_{1}$ and $\tau_{2}$ to develop a resonance-like enhancement at finite $\omega$. This could be an interesting experimentally observable feature. For very strong magnetic fields $\kappa \mathbf{B} \rightarrow \infty$, the chiral anomaly-induced effects would be pushed to the UV, corresponding to early time effects, such as in [77].

In Fig. 4 we show two-point correlators $G^{\mathrm{T}, \mathrm{L}}$ for different choices of $\kappa \mathbf{B}$. However, it is difficult to appreciate the anomaly-induced effects from Fig. 4 because in the correlators they get mixed with non-anomalous ones. To illuminate $\kappa \mathbf{B}$-correction to $G^{\mathrm{L}}$, in Fig. 5 we plot the difference $\delta G^{\mathrm{L}}=G^{\mathrm{L}}-G^{\mathrm{T}}$. From these plots, the effect of the chiral anomaly on the induced vector current is seen more clearly. We again notice a remarkable relative enhancement at intermediate values of $\omega$.

\section{Conclusions}

In this paper we continued explorations of the chiral anomaly induced transport within a holographic model containing two $U(1)$ fields interacting via Chern-Simons terms. For a finite temperature system, we computed off-shell constitutive relations for the vector/axial currents responding to external electromagnetic fields.

When a static spatially inhomogeneous magnetic field is the only external field that is turned on, we showed that both the CME and the CSE get corrected by derivative terms; see (3) and (4). Within the derivative expansion, we analytically calculated corrections up to third order in the expansion; see (48) and (49) and (51) and (52). Apart from the derivative corrections to CME and CSE, the diffusion constant $\mathcal{D}_{0}$ was found to receive a negative anomaly-induced correction; see (6). The dispersion relation of the chiral magnetic wave was also found to be modified; see (7). 
In the second part of our study, we focussed on the case of time-varying electric and constant magnetic fields without any externally enforced axial charge asymmetry, though the $\vec{E}(t) \cdot \overrightarrow{\mathbf{B}}$ term in the continuity equation (59) generates the axial charge density $\rho_{5}$ (and thus $\mu_{5}$ ) dynamically. For such a configuration of the external fields, we first analysed the most general constitutive relations for the vector/axial currents; see (8) and (9). Then, within the derivative expansion, we explicitly calculated the currents up to third order at nonlinear level; see (65) and (66). When put on shell, the axial current $\vec{J}_{5}$ is fully nonlinear in the external electric field.

Employing another approximation, we linearised the constitutive relations assuming the electric field is weak (10). Within this approximation the axial current is zero, while the "off-shell" vector current is parameterised by three frequency-dependent transport coefficient functions: the electric conductivity $\sigma_{e}$, and two chiral anomaly-induced conductivities $\tau_{1}, \tau_{2}$; see (11). In the DC limit, we analytically computed these conductivities; see (88) and (89). Then, for generic $\omega$, the numerical plots were presented in Sect. 4.2. Based on these studies, we notice that the anomaly-induced effects get enhanced at some finite frequency $\omega$, whereas the position of the maximum and strength of the effect depends on the external magnetic field. It might be an effect worth looking for experimentally.

Acknowledgements We would like to thank Dmitri E. Kharzeev, Alex Kovner, Andrey Sadofyev, Derek Teaney, and Ho-Ung Yee for useful discussions related to this work. YB would like to thank KITPC (Beijing) for financial support and hospitality, Physics Department of the University of Connecticut for hospitality where part of this work was done. This work was supported by the ISRAELI SCIENCE FOUNDATION Grant \#1635/16, BSF Grant \#012124, the People Program (Marie Curie Actions) of the European Union's Seventh Framework under REA Grant agreement \#318921; and the Council for Higher Education of Israel under the PBC Program of Fellowships for Outstanding Post-doctoral Researchers from China and India (2015-2016).

Open Access This article is distributed under the terms of the Creative Commons Attribution 4.0 International License (http://creativecomm ons.org/licenses/by/4.0/), which permits unrestricted use, distribution, and reproduction in any medium, provided you give appropriate credit to the original author(s) and the source, provide a link to the Creative Commons license, and indicate if changes were made.

Funded by SCOAP ${ }^{3}$.

\section{Appendix A: Supplement for Sect. 3}

In this appendix we provide computational details regarding non-renormalisation of CME and its gradient corrections up to third order. The dynamical equations (37)-(40) have a special property: in all equations, the first two terms can be rewritten as total derivatives of $\mathbb{V}_{\mu}, \mathbb{A}_{\mu}$. Treating all the remaining terms in (37)-(40) as sources, (37)-(40) can be integrated over $r$ twice, resulting in the following integral forms:

$$
\begin{aligned}
\mathbb{V}_{t}(r)= & -\int_{\mathrm{r}}^{\infty} \frac{\mathrm{d} x}{x^{3}} \int_{x}^{\infty} \\
& \times\left\{y \partial_{y} \partial_{k} \mathbb{V}_{k}+12 \kappa \epsilon^{i j k}\left[\partial_{y} \mathbb{A}_{i}\left(\partial_{j} \mathcal{V}_{k}+\partial_{j} \mathbb{V}_{k}\right)\right.\right. \\
& \left.\left.+\partial_{y} \mathbb{V}_{i} \partial_{j} \mathbb{A}_{k}\right]\right\} \mathrm{d} y, \\
& \times \underline{r \rightarrow \infty} \mathcal{O}\left(\frac{\log r}{r^{3}}\right), \\
\mathbb{V}_{i}(r)= & \int_{\mathrm{r}}^{\infty} \frac{-x \mathrm{~d} x}{x^{4}-1} \\
& \times\left\{G_{i}(x)-\frac{1-x}{2 x} \partial_{i} \rho-\partial_{k} \mathcal{F}_{k i}^{V} \log x\right. \\
& \left.-12 \kappa B_{i}\left(\mu_{5}+\mathbb{A}_{t}-\frac{1}{2 x^{2}} \rho_{5}\right)\right\} \\
& \times \frac{r \rightarrow \infty}{\longrightarrow}-\frac{\partial_{i} \rho}{4 r^{2}}+\frac{1+2 \log r}{4 r^{2}} \partial_{k} \mathcal{F}_{k i}^{V}+\frac{6}{r^{2}} \kappa \mu_{5} B_{i} \\
& -\frac{1}{2 r^{2}} G_{i}(x=\infty)+\mathcal{O}\left(\frac{\log r}{r^{3}}\right), \\
\mathbb{A}_{t}(r)= & -\int_{\mathrm{r}}^{\infty} \frac{\mathrm{d} x}{x^{3}} \int_{x}^{\infty} \\
& \times\left\{y \partial_{y} \partial_{k} \mathbb{A}_{k}+12 \kappa \epsilon^{i j k}\left[\partial_{y} \mathbb{V}_{i}\left(\partial_{j} \mathcal{V}_{k}+\partial_{j} \mathbb{V}_{k}\right)\right.\right. \\
& \left.\left.+\partial_{y} \mathbb{A}_{i} \partial_{j} \mathbb{A}_{k}\right]\right\} \mathrm{d} y \\
& \times r \rightarrow \infty)
\end{aligned}
$$

where $\mu$ and $\mu_{5}$ are the chemical potentials defined in (36). The frame convention (35) was utilised to fix integration constants, one for $\mathbb{V}_{t}$ and one for $\mathbb{A}_{t}$. The functions $G_{i}(x)$ and $H_{i}(x)$ are

$$
\begin{aligned}
G_{i}(x)= & \int_{1}^{x} \mathrm{~d} y\left\{-2 y \partial_{y} \partial_{t} \mathbb{V}_{i}+y \partial_{y} \partial_{i} \mathbb{V}_{t}\right. \\
& -\left(\partial_{t} \mathbb{V}_{i}-\partial_{i} \mathbb{V}_{t}\right)-\frac{1}{y}\left(\partial^{2} \mathbb{V}_{i}-\partial_{i} \partial_{k} \mathbb{V}_{k}\right) \\
& -12 \kappa \epsilon^{i j k} \partial_{y}\left(\mathbb{A}_{t}-\frac{1}{2 y^{2}} \rho_{5}\right) \partial_{j} \mathbb{V}_{k} \\
& -12 \kappa \epsilon^{i j k} \partial_{y}\left(\mathbb{V}_{t}-\frac{1}{2 y^{2}} \rho\right) \partial_{j} \mathbb{A}_{k} \\
& +12 \kappa \epsilon^{i j k} \partial_{y} \mathbb{A}_{j}\left[\left(\partial_{t} \mathbb{V}_{k}-\partial_{k} \mathbb{V}_{t}\right)+\frac{1}{2 y^{2}} \partial_{k} \rho\right] \\
& +12 \kappa \epsilon^{i j k} \partial_{y} \mathbb{V}_{j} \\
& \left.\times\left[\left(\partial_{t} \mathbb{A}_{k}-\partial_{k} \mathbb{A}_{t}\right)+\frac{1}{2 y^{2}} \partial_{k} \rho_{5}\right]\right\},
\end{aligned}
$$




$$
\begin{aligned}
H_{i}(x)= & \int_{1}^{x} \mathrm{~d} y\left\{-2 y \partial_{y} \partial_{t} \mathbb{A}_{i}+y \partial_{y} \partial_{i} \mathbb{A}_{t}\right. \\
& -\left(\partial_{t} \mathbb{A}_{i}-\partial_{i} \mathbb{A}_{t}\right)-\frac{1}{y}\left(\partial^{2} \mathbb{A}_{i}-\partial_{i} \partial_{k} \mathbb{A}_{k}\right) \\
- & 12 \kappa \epsilon^{i j k} \partial_{y}\left(\mathbb{V}_{t}-\frac{1}{2 y^{2}} \rho\right) \partial_{j} \mathbb{V}_{k} \\
& -12 \kappa \epsilon^{i j k} \partial_{y}\left(\mathbb{A}_{t}-\frac{1}{2 y^{2}} \rho_{5}\right) \partial_{j} \mathbb{A}_{k} \\
+ & 12 \kappa \epsilon^{i j k} \partial_{y} \mathbb{V}_{j}\left[\left(\partial_{t} \mathbb{V}_{k}-\partial_{k} \mathbb{V}_{t}\right)+\frac{1}{2 y^{2}} \partial_{k} \rho\right] \\
& +12 \kappa \epsilon^{i j k} \partial_{y} \mathbb{A}_{j} \\
\times & {\left.\left[\left(\partial_{t} \mathbb{A}_{k}-\partial_{k} \mathbb{A}_{t}\right)+\frac{1}{2 y^{2}} \partial_{k} \rho_{5}\right]\right\} . }
\end{aligned}
$$

Although we were unable to solve (42)-(45) for generic $\rho, \rho_{5}, \vec{B}(\vec{x})$, integral forms (94)-(97) help to explore general forms of $J^{\mu} / J_{5}^{\mu}$, as quoted in (3) and (4).

In the hydrodynamic limit, we analytically solved the dynamical equations (42)-(45) within the boundary derivative expansion (5). $\mathbb{V}_{\mu}^{[n]}$ and $\mathbb{A}_{\mu}^{[n]}$ up to $n=2$ are listed below.

$\mathbb{V}_{t}^{[1]}=\mathbb{A}_{t}^{[1]}=0$,

$$
\begin{aligned}
\mathbb{V}_{i}^{[1]}= & -\frac{1}{8}\left[\log \frac{1+r^{2}}{(1+r)^{2}}-2 \arctan (r)+\pi\right] \partial_{i} \rho \\
& +3 \kappa \rho_{5} B_{i} \log \frac{1+r^{2}}{r^{2}}, \\
\mathbb{A}_{i}^{[1]}= & \mathbb{V}_{i}^{[1]}\left(\rho \leftrightarrow \rho_{5}\right), \\
\mathbb{V}_{t}^{[2]}= & -\int_{\mathrm{r}}^{\infty} \frac{\mathrm{d} x}{x^{3}} \int_{x}^{\infty} \mathrm{d} y \\
& \times\left\{\frac{y \partial^{2} \rho}{2\left(y^{2}+1\right)(y+1)}-\frac{y 6 \kappa B_{k} \partial_{k} \rho_{5}}{\left(y^{2}+1\right)(y+1)}-\frac{72 \kappa^{2}}{y\left(y^{2}+1\right)} \rho B^{2}\right\} \\
& \stackrel{r=1}{=}-\frac{1}{16}(\pi-2 \log 2) \partial^{2} \rho+\frac{3}{4}(\pi-2 \log 2) \\
& \times \kappa B_{k} \partial_{k} \rho_{5}-18(1-2 \log 2) \kappa^{2} \rho B^{2},
\end{aligned}
$$$$
\mathbb{A}_{t}^{[2]}=\mathbb{V}_{t}^{[2]}\left(\rho \leftrightarrow \rho_{5}\right),
$$$$
\mathbb{V}_{i}^{[2]}=b_{0} \partial_{k} \mathcal{F}_{k i}^{V}+b_{1} \partial_{t} \partial_{i} \rho+b_{2} 6 \kappa \partial_{t} \rho_{5} B_{i}+b_{3} 36 \kappa^{2} \epsilon^{i j k}
$$$$
\times\left[\rho_{5} \partial_{j}\left(\rho_{5} B_{k}\right)+\rho \partial_{j}\left(\rho B_{k}\right)\right]
$$$$
+b_{4} 36 \kappa^{2} \epsilon^{i j k}\left(\rho B_{j} \partial_{k} \rho+\rho_{5} B_{j} \partial_{k} \rho_{5}\right) \text {, }
$$

$$
\begin{aligned}
\mathbb{A}_{i}^{[2]}= & b_{1} \partial_{t} \partial_{i} \rho_{5}+b_{2} 6 \kappa \partial_{t} \rho B_{i}+b_{3} 36 \kappa^{2} \epsilon^{i j k} \\
& \times\left[\rho \partial_{j}\left(\rho_{5} B_{k}\right)+\rho_{5} \partial_{j}\left(\rho B_{k}\right)\right]+b_{4} 36 \kappa^{2} \epsilon^{i j k} \\
& \times\left(\rho_{5} B_{j} \partial_{k} \rho+\rho B_{j} \partial_{k} \rho_{5}\right),
\end{aligned}
$$

where

$b_{0}=\int_{\mathrm{r}}^{\infty} \frac{x \mathrm{~d} x}{x^{4}-1} \int_{1}^{x} \frac{\mathrm{d} y}{y}$,

$$
\begin{aligned}
b_{1}= & \int_{\mathrm{r}}^{\infty} \frac{x \mathrm{~d} x}{x^{4}-1} \int_{1}^{x} \mathrm{~d} y \\
& \times\left\{\frac{y}{\left(y^{2}+1\right)(y+1)}\right. \\
& \left.\quad-\frac{1}{8}\left[\log \frac{1+y^{2}}{(1+y)^{2}}-2 \arctan (y)+\pi\right]\right\}, \\
b_{2}= & \int_{\mathrm{r}}^{\infty} \frac{x \mathrm{~d} x}{x^{4}-1} \int_{1}^{x} \mathrm{~d} y\left\{-\frac{2}{y^{2}+1}+\frac{1}{2} \log \frac{1+y^{2}}{y^{2}}\right\},
\end{aligned}
$$

$b_{3}=\int_{\mathrm{r}}^{\infty} \frac{x \mathrm{~d} x}{x^{4}-1} \int_{1}^{x} \frac{1}{y^{3}} \log \frac{1+y^{2}}{y^{2}} \mathrm{~d} y$,

$b_{4}=\int_{\mathrm{r}}^{\infty} \frac{x \mathrm{~d} x}{x^{4}-1} \int_{1}^{x} \frac{\mathrm{d} y}{y^{3}\left(y^{2}+1\right)}$.

These perturbative solutions, once inserted into (98), (99) and (36), produces the results (48)-(52).

\section{Appendix B: Supplement for Sect. 4}

This appendix contains calculational details for Sect. 4. As explained in Appendix 1, integrating the dynamical equations (61)-(64) over $r$ twice results in the following integral forms:

$$
\begin{aligned}
\mathbb{V}_{t}= & 12 \kappa \int_{\mathrm{r}}^{\infty} \frac{\mathrm{d} x}{x^{3}} \mathbb{A}_{k} \mathbf{B}_{k} \stackrel{r \rightarrow \infty}{\longrightarrow} \mathcal{O}\left(\frac{1}{r^{3}}\right), \\
\mathbb{V}_{i}= & \int_{\mathrm{r}}^{\infty} \frac{x \mathrm{~d} x}{x^{4}-1} \int_{1}^{x} \mathrm{~d} y\left\{2 y \partial_{y} \partial_{t} \mathbb{V}_{i}+\partial_{t} \mathbb{V}_{i}-E_{i}\right. \\
& +12 \kappa \partial_{y}\left(\mathbb{A}_{t}-\frac{1}{2 y^{2}} \rho_{5}\right) \mathbf{B}_{i} \\
& \left.-12 \kappa \epsilon^{i j k} \partial_{y} \mathbb{A}_{j}\left(-E_{k}+\partial_{t} \mathbb{V}_{k}\right)-12 \kappa \epsilon^{i j k} \partial_{y} \mathbb{V}_{j} \partial_{t} \mathbb{A}_{k}\right\} \\
& \stackrel{r \rightarrow \infty}{\rightarrow} \frac{1+2 \log r}{4 r^{2}} \partial_{t} E_{i}-\left(\frac{1}{r}-\frac{1}{2 r^{2}}\right) E_{i}+\frac{6}{r^{2}} \kappa \mu_{5} \mathbf{B}_{i} \\
& -\frac{6}{r^{2}} \kappa \epsilon^{i j k} \mathbb{A}_{j}(1) E_{k} \\
& +\frac{1}{2 r^{2}} \bar{G}_{i}(x=\infty)+\mathcal{O}\left(\frac{1}{r^{3}}\right),
\end{aligned}
$$

$$
\begin{aligned}
\mathbb{A}_{t}= & 12 \kappa \int_{\mathrm{r}}^{\infty} \frac{\mathrm{d} x}{x^{3}} \mathbb{V}_{k} \mathbf{B}_{k} \stackrel{r \rightarrow \infty}{\longrightarrow} \mathcal{O}\left(\frac{1}{r^{3}}\right) \\
\mathbb{A}_{i}= & \int_{\mathrm{r}}^{\infty} \frac{x \mathrm{~d} x}{x^{4}-1} \int_{1}^{x} \mathrm{~d} y\left\{2 y \partial_{y} \partial_{t} \mathbb{A}_{i}+\partial_{t} \mathbb{A}_{i}+12 \kappa \partial_{y} \mathbb{V}_{t} \mathbf{B}_{i}\right. \\
& +12 \kappa \epsilon^{i j k} \partial_{y} \mathbb{V}_{j} E_{k} \\
& \left.-12 \kappa \epsilon^{i j k} \partial_{y} \mathbb{V}_{j} \partial_{t} \mathbb{V}_{k}-12 \kappa \epsilon^{i j k} \partial_{y} \mathbb{A}_{j} \partial_{t} \mathbb{A}_{k}\right\} \\
& \stackrel{r \rightarrow \infty}{\frac{6}{r^{2}} \kappa \mu \mathbf{B}_{i}-\frac{6}{r^{2}} \kappa \epsilon^{i j k} \mathbb{V}_{j}(1) E_{k}} \\
& +\frac{1}{2 r^{2}} \bar{H}_{i}(x=\infty)+\mathcal{O}\left(\frac{1}{r^{3}}\right)
\end{aligned}
$$


where $\mu, \mu_{5}$ are defined via (36). $\bar{G}_{i}$ and $\bar{H}_{i}$ are

$$
\begin{aligned}
\bar{G}_{i}(x)= & \int_{1}^{x} \mathrm{~d} y\left\{2 y \partial_{y} \partial_{t} \overline{\mathbb{V}}_{i}+\partial_{t} \overline{\mathbb{V}}_{i}\right. \\
& \left.-12 \kappa \epsilon^{i j k}\left(\partial_{y} \mathbb{A}_{j} \partial_{t} \mathbb{V}_{k}+\partial_{y} \mathbb{V}_{j} \partial_{t} \mathbb{A}_{k}\right)\right\}, \\
\bar{H}_{i}(x)= & \int_{1}^{x} \mathrm{~d} y\left\{2 y \partial_{y} \partial_{t} \mathbb{A}_{i}+\partial_{t} \mathbb{A}_{i}\right. \\
& \left.-12 \kappa \epsilon^{i j k}\left(\partial_{y} \mathbb{V}_{j} \partial_{t} \mathbb{V}_{k}+\partial_{y} \mathbb{A}_{j} \partial_{t} \mathbb{A}_{k}\right)\right\},
\end{aligned}
$$

where $\overline{\mathbb{V}}_{i}=\mathbb{V}_{i}+E_{i} / r$. Note that we have split the $E_{i} / r$ piece from $\mathbb{V}_{i}$ so that $\bar{G}_{i}(x)$ is well defined at $x=\infty$. Via the general formulas (29), the large $r$ behaviours (112)-(115) produce the formal results of (8) and (9).

Under the boundary derivative expansion (5), (70)-(73) can be solved perturbatively. Up to second order $\mathcal{O}\left(\partial^{2}\right)$, the corrections $\mathbb{V}_{\mu}$ and $\mathbb{A}_{\mu}$ are

$$
\mathbb{V}_{t}=\mathcal{O}\left(\partial^{3}\right)
$$

$$
\begin{aligned}
\mathbb{A}_{t}= & a_{0}(r) 12 \kappa \vec{E} \cdot \overrightarrow{\mathbf{B}}-\frac{18}{r^{2}}\left[1-\left(1+r^{2}\right) \log \frac{1+r^{2}}{r^{2}}\right] \kappa^{2} \rho_{5} \mathbf{B}^{2} \\
& +\mathcal{O}\left(\partial^{3}\right) \\
\mathbb{V}_{i}= & -\frac{1}{4}\left[\log \frac{(1+r)^{2}}{1+r^{2}}-2 \arctan (r)+\pi\right] E_{i} \\
& +3 \log \frac{1+r^{2}}{r^{2}} \kappa \rho_{5} \mathbf{B}_{i}+a_{1}(r) \partial_{t} E_{i} \\
& +b_{2}(r) 6 \kappa \partial_{t} \rho_{5} \mathbf{B}_{i}+\mathcal{O}\left(\partial^{3}\right)
\end{aligned}
$$

$\mathbb{A}_{i}=a_{2}(r) 72 \kappa^{2} \rho_{5} \epsilon^{i j k} \mathbf{B}_{j} E_{k}+\mathcal{O}\left(\partial^{3}\right)$,

where

$$
\begin{gathered}
a_{0}(r)=-\frac{1}{8 r^{2}}\left\{\left(r^{2}+1\right)\left(2 \operatorname{arccot}(r)-\log \frac{1+r^{2}}{r^{2}}\right)\right. \\
\left.+2\left(r^{2}-1\right) \log \frac{r}{1+r}\right\}
\end{gathered}
$$

$$
\begin{aligned}
a_{1}(r)= & -\int_{\mathrm{r}}^{\infty} \frac{x \mathrm{~d} x}{x^{4}-1} \int_{1}^{x} \mathrm{~d} y \\
& \times\left\{-\frac{2 y^{2}}{1+y^{2}}+\frac{1}{4}\left[\log \frac{(1+y)^{2}}{1+y^{2}}-2 \arctan (y)+\pi\right]\right\},
\end{aligned}
$$

$a_{2}(r)=-\int_{\mathrm{r}}^{\infty} \frac{x \mathrm{~d} x}{x^{4}-1} \int_{1}^{x} \frac{\mathrm{d} y}{y\left(y^{2}+1\right)}$,

and $b_{2}$ is presented in (109). From these perturbative results, we deduce the hydro expansion for the currents $J^{\mu} / J_{5}^{\mu}$ and chemical potentials $\mu / \mu_{5}$, as summarised in (65), (66) and (68).

\section{References}

1. L.D. Landau, E.M. Lifshitz, Fluid Mechanics: Course of Theoretical Physics, vol. 6. (Butterworth-Heinemann, Oxford, 1965)

2. D. Forster, Hydrodynamic Fluctuations, Broken Symmetry, and Correlation Functions. (Westview Press, Boulder, 1995)

3. I. Muller, Zum Paradoxon der Warmeleitungstheorie. Z. Phys. 198, 329-344 (1967). doi:10.1007/BF01326412

4. W. Israel, Nonstationary irreversible thermodynamics: a causal relativistic theory. Ann. Phys. 100, 310-331 (1976). doi:10.1016/ 0003-4916(76)90064-6

5. W. Israel, J. Stewart, Thermodynamics of nonstationary and transient effects in a relativistic gas. Phys. Lett. A 58, 213-215 (1976). doi:10.1016/0375-9601(76)90075-X

6. W. Israel, J. Stewart, Transient relativistic thermodynamics and kinetic theory. Ann. Phys. 118, 341-372 (1979). doi:10.1016/ 0003-4916(79)90130-1

7. Y. Bu, M. Lublinsky, Linearly resummed hydrodynamics in a weakly curved spacetime. JHEP 04, 136 (2015). doi:10.1007/ JHEP04(2015)136. arXiv:1502.08044 [hep-th]

8. L.P. Kadanoff, P.C. Martin, Hydrodynamic equations and correlation functions. Ann. Phys. 24, 419-469 (1963). doi:10.1016/ 0003-4916(63)90078-2

9. Y. Bu, M. Lublinsky, A. Sharon, $U(1)$ current from the AdS/CFT: diffusion, conductivity and causality. JHEP 04, 136 (2016). doi:10. 1007/JHEP04(2016)136. arXiv:1511.08789 [hep-th]

10. Y. Bu, M. Lublinsky, All order linearized hydrodynamics from fluid-gravity correspondence. Phys. Rev. D 90(8), 086003 (2014). doi:10.1103/PhysRevD.90.086003. arXiv:1406.7222 [hep-th]

11. Y. Bu, M. Lublinsky, Linearized fluid/gravity correspondence: from shear viscosity to all order hydrodynamics. JHEP 11, 064 (2014). doi:10.1007/JHEP11(2014)064. arXiv:1409.3095 [hep-th]

12. Y. Bu, M. Lublinsky, A. Sharon, Hydrodynamics dual to EinsteinGauss-Bonnet gravity: all-order gradient resummation. JHEP 06, 162 (2015). doi:10.1007/JHEP06(2015)162. arXiv:1504.01370 [hep-th]

13. M.P. Heller, R.A. Janik, P. Witaszczyk, Hydrodynamic gradient expansion in gauge theory plasmas. Phys. Rev. Lett. 110(21), 211602 (2013). doi:10.1103/PhysRevLett.110.211602. arXiv:1302.0697 [hep-th]

14. S. Bhattacharyya, V.E. Hubeny, S. Minwalla, M. Rangamani, Nonlinear fluid dynamics from gravity. JHEP 02, 045 (2008). doi:10. 1088/1126-6708/2008/02/045. arXiv:0712.2456 [hep-th]

15. D. Kharzeev, Parity violation in hot QCD: why it can happen, and how to look for it. Phys. Lett. B633, 260-264 (2006). doi:10.1016/ j.physletb.2005.11.075. arXiv:hep-ph/0406125 [hep-ph]

16. D. Kharzeev, A. Zhitnitsky, Charge separation induced by P-odd bubbles in QCD matter. Nucl. Phys. A 797, 67-79 (2007). doi:10. 1016/j.nuclphysa.2007.10.001. arXiv:0706.1026 [hep-ph]

17. D.E. Kharzeev, L.D. McLerran, H.J. Warringa, The Effects of topological charge change in heavy ion collisions: event by event $\mathrm{P}$ and CP violation. Nucl. Phys. A 803, 227-253 (2008). doi:10.1016/j. nuclphysa.2008.02.298. arXiv:0711.0950 [hep-ph]

18. K. Fukushima, D.E. Kharzeev, H.J. Warringa, The chiral magnetic effect. Phys. Rev. D 78, 074033 (2008). doi:10.1103/PhysRevD. 78.074033. arXiv:0808.3382 [hep-ph]

19. D.E. Kharzeev, Topologically induced local $P$ and $C P$ violation in QCD $\times$ QED. Ann. Phys. 325, 205-218 (2010). doi:10.1016/j.aop. 2009.11.002. arXiv:0911.3715 [hep-ph]

20. A. Vilenkin, Equilibrium parity violating current in a magnetic field. Phys. Rev. D 22, 3080-3084 (1980). doi:10.1103/PhysRevD. 22.3080

21. M. Giovannini, M.E. Shaposhnikov, Primordial hypermagnetic fields and triangle anomaly. Phys. Rev. D 57, 2186-2206 (1998). doi:10.1103/PhysRevD.57.2186. arXiv:hep-ph/9710234 [hep-ph] 
22. A. Yu. Alekseev, V.V. Cheianov, J. Frohlich, Universality of transport properties in equilibrium, Goldstone theorem and chiral anomaly. Phys. Rev. Lett. 81, 3503-3506 (1998). doi:10.1103/ PhysRevLett.81.3503. arXiv:cond-mat/9803346 [cond-mat]

23. D.E. Kharzeev, H.J. Warringa, Chiral magnetic conductivity. Phys. Rev. D 80, 034028 (2009). doi:10.1103/PhysRevD.80.034028. arXiv:0907.5007 [hep-ph]

24. K. Fukushima, D.E. Kharzeev, H.J. Warringa, Real-time dynamics of the chiral magnetic effect. Phys. Rev. Lett. 104, 212001 (2010). doi:10.1103/PhysRevLett.104.212001. arXiv:1002.2495 [hep-ph]

25. A.V. Sadofyev, V.I. Shevchenko, V.I. Zakharov, Notes on chiral hydrodynamics within effective theory approach. Phys. Rev. D 83, 105025 (2011). doi:10.1103/PhysRevD.83.105025. arXiv:1012.1958 [hep-th]

26. D. Satow, H.-U. Yee, Chiral magnetic effect at weak coupling with relaxation dynamics. Phys. Rev. D 90(1), 014027 (2014). doi:10. 1103/PhysRevD.90.014027. arXiv:1406.1150 [hep-ph]

27. H.-U. Yee, Chiral magnetic and vortical effects in higher dimensions at weak coupling. Phys. Rev. D 90(6), 065021 (2014). doi:10. 1103/PhysRevD.90.065021. arXiv:1406.3584 [hep-th]

28. A. Jimenez-Alba, H.-U. Yee, Second order transport coefficient from the chiral anomaly at weak coupling: diagrammatic resummation. Phys. Rev. D 92(1), 014023 (2015). doi:10.1103/PhysRevD. 92.014023. arXiv:1504.05866 [hep-ph]

29. S.D. Chowdhury, J.R. David, Anomalous transport at weak coupling. JHEP 11, 048 (2015). doi:10.1007/JHEP11(2015)048. arXiv:1508.01608 [hep-th]

30. P.V. Buividovich, M.N. Chernodub, E.V. Luschevskaya, M.I. Polikarpov, Numerical evidence of chiral magnetic effect in lattice gauge theory. Phys. Rev. D 80, 054503 (2009). doi:10.1103/ PhysRevD.80.054503. arXiv:0907.0494 [hep-lat]

31. M. Abramczyk, T. Blum, G. Petropoulos, R. Zhou, Chiral magnetic effect in 2+1 flavor QCD+QED. PoS LAT 2009, 181 (2009). arXiv:0911.1348 [hep-lat]

32. K. Fukushima, D.E. Kharzeev, H.J. Warringa, Electric-current susceptibility and the chiral magnetic effect. Nucl. Phys. A 836, 311-336 (2010). doi:10.1016/j.nuclphysa.2010.02.003. arXiv:0912.2961 [hep-ph]

33. V.V. Braguta, P.V. Buividovich, T. Kalaydzhyan, S.V. Kuznetsov, M.I. Polikarpov, The chiral magnetic effect and chiral symmetry breaking in SU(3) quenched lattice gauge theory. Phys. Atom. Nucl. 75, 488-492 (2012). doi:10.1134/S1063778812030052. arXiv:1011.3795 [hep-lat]

34. A. Yamamoto, Chiral magnetic effect in lattice QCD with a chiral chemical potential. Phys. Rev. Lett. 107, 031601 (2011). doi:10. 1103/PhysRevLett.107.031601. arXiv:1105.0385 [hep-lat]

35. V. Braguta, M.N. Chernodub, K. Landsteiner, M.I. Polikarpov, M.V. Ulybyshev, Numerical evidence of the axial magnetic effect. Phys. Rev. D 88, 071501 (2013). doi:10.1103/PhysRevD.88. 071501. arXiv:1303.6266 [hep-lat]

36. H.-U. Yee, Holographic chiral magnetic conductivity. JHEP 11, 085 (2009). doi:10.1088/1126-6708/2009/11/085. arXiv:0908.4189 [hep-th]

37. A. Rebhan, A. Schmitt, S.A. Stricker, Anomalies and the chiral magnetic effect in the Sakai-Sugimoto model. JHEP 01, 026 (2010). doi:10.1007/JHEP01(2010)026. arXiv:0909.4782 [hep-th]

38. D.T. Son, P. Surowka, Hydrodynamics with triangle anomalies. Phys. Rev. Lett. 103, 191601 (2009). doi:10.1103/PhysRevLett. 103.191601. arXiv:0906.5044 [hep-th]

39. Y. Matsuo, S.-J. Sin, S. Takeuchi, T. Tsukioka, Magnetic conductivity and Chern-Simons term in holographic hydrodynamics of charged AdS black hole. JHEP 04, 071 (2010). doi:10.1007/ JHEP04(2010)071. arXiv:0910.3722 [hep-th]

40. A. Gorsky, P.N. Kopnin, A.V. Zayakin, On the chiral magnetic effect in soft-wall AdS/QCD. Phys. Rev. D 83, 014023 (2011). doi:10.1103/PhysRevD.83.014023. arXiv:1003.2293 [hep-ph]
41. V.A. Rubakov, On chiral magnetic effect and holography. arXiv: 1005.1888 [hep-ph]

42. A. Gynther, K. Landsteiner, F. Pena-Benitez, A. Rebhan, Holographic anomalous conductivities and the chiral magnetic effect. JHEP 02, 110 (2011). doi:10.1007/JHEP02(2011)110. arXiv:1005.2587 [hep-th]

43. T. Kalaydzhyan, I. Kirsch, Fluid/gravity model for the chiral magnetic effect. Phys. Rev. Lett. 106, 211601 (2011). doi:10.1103/ PhysRevLett.106.211601. arXiv:1102.4334 [hep-th]

44. Y.-P. Hu, P. Sun, J.-H. Zhang, Hydrodynamics with conserved current via AdS/CFT correspondence in the Maxwell-Gauss-Bonnet gravity. Phys. Rev. D 83, 126003 (2011). doi:10.1103/PhysRevD. 83.126003. arXiv: 1103.3773 [hep-th]

45. Y.-P. Hu, C. Park, Chern-Simons effect on the dual hydrodynamics in the Maxwell-Gauss-Bonnet gravity. Phys. Lett. B 714, 324330 (2012). doi:10.1016/j.physletb.2012.07.008. arXiv:1112.4227 [hep-th]

46. X. Bai, Y.-P. Hu, B.-H. Lee, Y.-L. Zhang, Holographic charged fluid with anomalous current at finite cutoff surface in Einstein-Maxwell gravity. JHEP 11, 054 (2012). doi:10.1007/JHEP11(2012)054. arXiv:1207.5309 [hep-th]

47. S. Lin, H.-U. Yee, Out-of-equilibrium chiral magnetic effect at strong coupling. Phys. Rev. D 88(2), 025030 (2013). doi:10.1103/ PhysRevD.88.025030. arXiv:1305.3949 [hep-ph]

48. U. Gursoy, A. Jansen, (Non)renormalization of anomalous conductivities and holography. JHEP 10, 092 (2014). doi:10.1007/ JHEP10(2014)092. arXiv:1407.3282 [hep-th]

49. U. Gursoy, J. Tarrio, Horizon universality and anomalous conductivities. JHEP 10, 058 (2015). doi:10.1007/JHEP10(2015)058. arXiv: 1410.1306 [hep-th]

50. S. Grozdanov, N. Poovuttikul, Universality of anomalous conductivities in theories with higher-derivative holographic duals. JHEP 09, 046 (2016). doi:10.1007/JHEP09(2016)046. arXiv: 1603.08770 [hep-th]

51. J.M. Maldacena, The large $\mathrm{N}$ limit of superconformal field theories and supergravity. Int. J. Theor. Phys. 38, 1113-1133 (1999). doi:10. 1023/A:1026654312961. arXiv:hep-th/9711200 [hep-th]. (Adv. Theor. Math. Phys. 2, 231 (1998))

52. S.S. Gubser, I.R. Klebanov, A.M. Polyakov, Gauge theory correlators from noncritical string theory. Phys. Lett. B 428, 105-114 (1998). doi:10.1016/S0370-2693(98)00377-3. arXiv:hep-th/9802109 [hep-th]

53. E. Witten, Anti-de Sitter space and holography. Adv. Theor. Math. Phys. 2, 253-291 (1998). arXiv:hep-th/9802150 [hep-th]

54. D.T. Son, A.R. Zhitnitsky, Quantum anomalies in dense matter. Phys. Rev. D 70, 074018 (2004). doi:10.1103/PhysRevD.70. 074018. arXiv:hep-ph/0405216 [hep-ph]

55. M.A. Metlitski, A.R. Zhitnitsky, Anomalous axion interactions and topological currents in dense matter. Phys. Rev. D 72, 045011 (2005). doi:10.1103/PhysRevD.72.045011. arXiv:hep-ph/0505072 [hep-ph]

56. X.G. Huang, J. Liao, Axial current generation from electric field: chiral electric separation effect. Phys. Rev. Lett. 110(23), 232-302 (2013). doi:10.1103/PhysRevLett.110.232302. arXiv:1303.7192 [nucl-th]

57. Y. Jiang, X.-G. Huang, J. Liao, Chiral electric separation effect in the quark-gluon plasma. Phys. Rev. D 91(4), 045001 (2015). doi:10.1103/PhysRevD.91.045001. arXiv:1409.6395 [nucl-th]

58. B. Keren-Zur, Y. Oz, Hydrodynamics and the detection of the QCD axial anomaly in heavy ion collisions. JHEP 06, 006 (2010). doi: 10. 1007/JHEP06(2010)006. arXiv:1002.0804 [hep-ph]

59. D.E. Kharzeev, D.T. Son, Testing the chiral magnetic and chiral vortical effects in heavy ion collisions. Phys. Rev. Lett. 106, 062301 (2011). doi:10.1103/PhysRevLett.106.062301. arXiv:1010.0038 [hep-ph] 
60. Y. Burnier, D.E. Kharzeev, J. Liao, H.-U. Yee, Chiral magnetic wave at finite baryon density and the electric quadrupole moment of quark-gluon plasma in heavy ion collisions. Phys. Rev. Lett. 107, 052303 (2011). doi:10.1103/PhysRevLett.107.052303. arXiv:1103.1307 [hep-ph]

61. H.U. Yee, Y. Yin, Realistic implementation of chiral magnetic wave in heavy ion collisions. Phys. Rev. C 89(4), 044909 (2014). doi:10. 1103/PhysRevC.89.044909. arXiv:1311.2574 [nucl-th]

62. V. Skokov, P. Sorensen, V. Koch, S. Schlichting, J. Thomas, S. Voloshin, G. Wang, H.-U. Yee, Chiral magnetic effect task force report. arXiv:1608.00982 [nucl-th]

63. D.E. Kharzeev, K. Landsteiner, A. Schmitt, H.-U. Yee, 'Strongly interacting matter in magnetic fields': an overview. Lect. Notes Phys. 871, 1-11 (2013). doi:10.1007/978-3-642-37305-3_1. arXiv:1211.6245 [hep-ph]

64. V.I. Zakharov, Chiral Magnetic effect in hydrodynamic approximation. Lect. Notes Phys. 871, 295 (2013). arXiv:1210.2186 [hep-ph]

65. D.E. Kharzeev, The chiral magnetic effect and anomaly-induced transport. Prog. Part. Nucl. Phys. 75, 133-151 (2014). doi:10.1016/ j.ppnp.2014.01.002. arXiv:1312.3348 [hep-ph]

66. X.-G. Huang, Electromagnetic fields and anomalous transports in heavy-ion collisions-a pedagogical review. Rept. Prog. Phys. 79(7), 076302 (2016). doi:10.1088/0034-4885/79/7/ 076302. arXiv:1509.04073 [nucl-th]

67. D.E. Kharzeev, J. Liao, S.A. Voloshin, G. Wang, Chiral magnetic and vortical effects in high-energy nuclear collisions - a status report. Prog. Part. Nucl. Phys. 88, 1-28 (2016). doi:10.1016/j.ppnp. 2016.01.001. arXiv:1511.04050 [hep-ph]

68. Y. Bu, M. Lublinsky, A. Sharon, Anomalous transport from holography: part I. JHEP 11, 093 (2016). doi:10.1007/ JHEP11(2016)093. arXiv:1608.08595 [hep-th]

69. T. Sakai, S. Sugimoto, Low energy hadron physics in holographic QCD. Prog. Theor. Phys. 113, 843-882 (2005). doi:10.1143/PTP. 113.843. arXiv:hep-th/0412141 [hep-th]

70. D.E. Kharzeev, H.-U. Yee, Chiral magnetic wave. Phys. Rev. D 83, 085007 (2011). doi:10.1103/PhysRevD.83.085007. arXiv: 1012.6026 [hep-th]

71. A.V. Sadofyev, M.V. Isachenkov, The chiral magnetic effect in hydrodynamical approach. Phys. Lett. B 697, 404-406 (2011). doi:10.1016/j.physletb.2011.02.041. arXiv:1010.1550 [hep-th]

72. P. Kovtun, A. Ritz, Universal conductivity and central charges. Phys. Rev. D 78, 066009 (2008). doi:10.1103/PhysRevD.78. 066009. arXiv:0806.0110 [hep-th]
73. A. Jimenez-Alba, K. Landsteiner, L. Melgar, Anomalous magnetoresponse and the Stückelberg axion in holography. Phys. Rev. D 90, 126004 (2014). doi:10.1103/PhysRevD.90.126004. arXiv:1407.8162 [hep-th]

74. K. Landsteiner, Y. Liu, Y.-W. Sun, Negative magnetoresistivity in chiral fluids and holography. JHEP 03, 127 (2015). doi:10.1007/ JHEP03(2015)127. arXiv:1410.6399 [hep-th]

75. A. Jimenez-Alba, K. Landsteiner, Y. Liu, Y.-W. Sun, Anomalous magnetoconductivity and relaxation times in holography. JHEP 07, 117 (2015). doi:10.1007/JHEP07(2015)117. arXiv:1504.06566 [hep-th]

76. Y.-W. Sun, Q. Yang, Negative magnetoresistivity in holography. JHEP 09, 122 (2016). doi:10.1007/JHEP09(2016)122. arXiv: 1603.02624 [hep-th]

77. M. Ammon, S. Grieninger, A. Jimenez-Alba, R.P. Macedo, L. Melgar, Holographic quenches and anomalous transport. JHEP 09, 131 (2016). doi:10.1007/JHEP09(2016)131. arXiv:1607.06817 [hepth]

78. H.B. Nielsen, M. Ninomiya, Adler-Bell-Jackiw anomaly and weyl fermions in crystal. Phys. Lett. B 130, 389-396 (1983). doi:10. 1016/0370-2693(83)91529-0

79. D.T. Son, B.Z. Spivak, Chiral anomaly and classical negative magnetoresistance of Weyl metals. Phys. Rev. B 88, 104412 (2013). doi:10.1103/PhysRevB.88.104412. arXiv:1206.1627 [cond-mat.mes-hall]

80. H.-J. Kim, K.-S. Kim, J.F. Wang, M. Sasaki, N. Satoh, A. Ohnishi, M. Kitaura, M. Yang, L. Li, Dirac versus Weyl fermions in topological insulators: Adler-Bell-Jackiw anomaly in transport phenomena. Phys. Rev. Lett. 111(24), 246603 (2013). doi:10.1103/ PhysRevLett.111.246603. arXiv:1307.6990 [cond-mat.str-el]

81. E.V. Gorbar, V.A. Miransky, I.A. Shovkovy, Chiral anomaly, dimensional reduction, and magnetoresistivity of Weyl and Dirac semimetals. Phys. Rev. B89(8), 085126 (2014). doi:10.1103/ PhysRevB.89.085126. arXiv:1312.0027 [cond-mat.mes-hall]

82. X. Huang et al., Observation of the chiral-anomaly-induced negative magnetoresistance in 3D Weyl semimetal TaAs. Phys. Rev. X. 5(3), 031023 (2015). doi:10.1103/PhysRevX.5.031023

83. H. Li, H. He, H.-Z. Lu, H. Zhang, H. Liu, R. Ma, Z. Fan, S.-Q. Shen, J. Wang, Negative magnetoresistance in dirac semimetal cd3as2. Nature Commun. 7, 10301 (2016). doi:10.1038/ncomms 10301 